\title{
NECESSARY AND SUFFICIENT CONDITIONS FOR THE REPRESENTATION OF A FUNCTION AS \\ A LAPLACE INTEGRAL*
}

BY

D. V. WIDDER

1. Introduction. In a previous paper $\dagger$ in these Transactions the author studied the singularities of functions defined by integrals of the form

$$
f(x)=\int_{0}^{\infty} e^{-x t} d \alpha(t),
$$

considering such an integral as a generalization of a Taylor series. All developments of that paper were on the assumption that $f(x)$ permitted of the integral representation (1.1). We wish to study here conditions on $f(x)$, both necessary and sufficient, for the validity of such representation. Following the analogy of Taylor's series we might at first be tempted to suppose that the analyticity of $f(x)$ in a half-plane, the region of convergence of an integral (1.1), would be the condition required. That this is not the case we see at once by recalling that such a function as $\sin x$, analytic in the entire plane, admits of no representation $\ddagger$ of the form (1.1).

We are led, however, to a correct conjecture by considering our problem as the analogue of the moment problem of $F$. Hausdorff. $\$$ This is the problem of determining a function $\chi(x)$ bounded and non-decreasing in the interval $0 \leqq x \leqq 1$ and such that

$$
\mu_{k}=\int_{0}^{1} x^{k} d \chi(x) \quad(k=0,1,2, \cdots) .
$$

Hausdorff has shown that the problem has a solution if and only if the sequence $\mu_{0}, \mu_{1}, \mu_{2}, \cdots$ is completely monotonic ("total monotone"). That is, the differences

$$
(-1)^{n} \Delta^{n} \mu_{m}=\mu_{m}-\left(\begin{array}{l}
n \\
1
\end{array}\right) \mu_{m+1}+\left(\begin{array}{l}
n \\
2
\end{array}\right) \mu_{m+2}-\cdots+(-1)^{n} \mu_{m+n}
$$

* Presented to the Society, December 30, 1930; received by the editors December 11, 1931.

$\dagger \mathrm{D}$. V. Widder, A generalization of Dirichlet's series and of Laplace's integrals by means of a Stieltjes integral, these Transactions, vol. 31 (1929), p. 694.

$\ddagger$ This follows at once from a result of $M$. Lerch, Sur un point de la theorie des fonctions generatrices d'Abel, Acta Mathematica, vol. 27 (1903), p. 339.

\& Felix Hausdorf, Momentprobleme für ein endliches Intervall, Mathematische Zeitschrift, vol. 16 (1923), p. 220. 
satisfy the inequalities

$$
(-1)^{n} \Delta^{n} \mu_{m} \geqq 0 \quad(n=0,1,2, \cdots ; m=0,1,2, \cdots) .
$$

If we generalize this moment problem by allowing $k$ to run through a continuous set of values, we are led to the integral equation

$$
\mu(y)=\int_{0}^{1} x^{y} d \chi(x)
$$

for the determination of a non-decreasing function $\chi(x)$. If we set $x=e^{-t}$, this equation becomes

where

$$
\mu(y)=\int_{0}^{\infty} e^{-y t} d \alpha(t)
$$

$$
\alpha(t)=-\chi\left(e^{-t}\right) .
$$

If $\alpha(t)$ is a non-decreasing function of $t$, then $\chi(x)$ will be a non-decreasing function of $x$, so that we are now required to solve an integral equation of type (1.1) for a non-decreasing function $\alpha(t)$. From Hausdorff's results we should be led to conjecture that the equation has a solution of the type desired if and only if $f(x)$ has derivatives of all orders satisfying the inequalities

$$
(-1)^{n} \frac{d^{n}}{d x^{n}} f(x) \geqq 0 \quad(n=0,1,2, \cdots),
$$

and this is in fact the case. This fact was first proved by S. Bernstein* in 1929. The present paper begins with a proof of this theorem following methods quite different from those of Bernstein. The more general problem of determining a solution of (1.1) which is merely of bounded variation is then attacked. A necessary and sufficient condition on $f(x)$ to guarantee the existence of a function $\alpha(t)$ of bounded variation and making the integral absolutely convergent is then obtained. The corresponding problem for an integral of the form

$$
f(x)=\int_{0}^{\infty} e^{-x t} \phi(t) d t
$$

is then treated. It is found that this equation has a solution $\phi(t)$ which is bounded and integrable if and only if

$$
\left|f^{(n)}(x)\right| \leqq K n ! /(x-c)^{n+1} \quad(x>c),
$$

where $K$ is some constant.

* Serge Bernstein, Sur les fonctions absolument monotones, Acta Mathematica, vol. 52 (1929), p. 1. The author had completed the proof of this theorem a few months after the publication of Bernstein's paper without being aware of its existence. 
We are then able to solve a problem of considerable importance in the theory of Dirichlet's series. We obtain conditions on $f(x)$ in order that the integral equation (1.1) may have a step-function solution. We thus obtain a necessary and sufficient condition for the representation of $f(x)$ in a Dirichlet series.*

We then investigate the representation of a function $f(x)$ by an integral of the form

$$
f(x)=\int_{0}^{1} e^{x t} d \alpha(t)
$$

with $\alpha(t)$ a bounded non-decreasing function, and find that a necessary and sufficient condition for such representation is that the sequence of derivatives of $f(x)$ at a point $x_{0}$,

$$
f\left(x_{0}\right), f^{\prime}\left(x_{0}\right), f^{\prime \prime}\left(x_{0}\right), \cdots,
$$

should be completely monotonic. We then inquire what properties a sequence $a_{0}, a_{1}, a_{2}, \cdots$ must have in order that there may exist a completely monotonic function $f(x)$ satisfying the equations

$$
f(n)=a_{n} \quad(n=0,1,2, \cdots),
$$

and find that it is necessary for the sequence to be completely monotonic. A slight change in the condition makes it both necessary and sufficient. Combining this with the previous result we are led to infer that the generalized derivative of arbitrary order $\rho$ of $f(x)$,

$$
{ }_{-\infty} D_{x}^{\rho} f(x)=\frac{1}{\Gamma(1-\nu)} \int_{0}^{\infty} t^{-\nu} f^{(m+1)}(x-t) d t,[\rho]=m, \rho=m+\nu,
$$

is a completely monotonic function of $\rho$ for every $x$ if and only if $f(x)$ has the form (1.2). Here the generalized derivative is defined in a form slightly different from that given by Riemann, but it is shown that the form adopted is equally good as a generalization for the functions under consideration since it reduces to the ordinary derivative when $\rho$ is an integer.

Throughout most of the paper functions of the real variable are considered. In the last section, however, it is shown that this is no essential restriction in the case of certain of the theorems, and in particular in the case of the theorem regarding Dirichlet's series. Slight modifications are made to make the theorem applicable to functions of the complex variable.

* References to earlier attempts to find such conditions will be found in Mémorial des Sciences Mathématiques, Fascicule XVII, Théorie Générale des Séries de Dirichlet, by M. G. Valiron, p. 30. The referee has called the author's attention to the following paper: Th. Kaluza, Entwickelbarkeit von Funktionen in Dirichletsche Reihen, Mathematische Zeitschrift, vol. 28 (1928), p. 203. 
2. Completely monotonic functions and sequences. We begin with several definitions.

Definition 1. A function $f(x)$ is completely monotonic in the interval $c<x<\infty$ if it has derivatives of all orders in this interval and if the inequalities

$$
(-1)^{n} f^{(n)}(x) \geqq 0 \quad(n=0,1,2, \cdots)
$$

are satisfied there.

Definition 2. A function $f(x)$ is completely monotonic in the interval $c \leqq x<\infty$ if it is completely monotonic in the interval $c<x<\infty$ and if $f(c+0)$ $=f(c) \neq \infty$.

Definition 3. The set of constants $\mu_{0}, \mu_{1}, \mu_{2}, \cdots$ form a completely monotonic sequence if

$$
(-1)^{n} \Delta^{n} \mu_{m} \geqq 0 \quad(n=0,1,2, \cdots ; m=0,1,2, \cdots),
$$

where

$$
(-1)^{n} \Delta^{n} \mu_{m}=\mu_{m}-\left(\begin{array}{c}
n \\
1
\end{array}\right) \mu_{m+1}+\left(\begin{array}{l}
n \\
2
\end{array}\right) \mu_{m+2}-\cdots+(-1)^{n} \mu_{m+n} .
$$

We now prove

THEOREM 1. If $f(x)$ is completely monotonic in the interval $c<x<\infty$, and if $\delta$ is any positive constant, then the set of constants

$$
f(a), f(a+\delta), f(a+2 \delta), \cdots \quad(c<a<\infty)
$$

forms a completely monotonic sequence.

For, we have $\Delta^{n} f(a+m \delta)=f^{(n)}(\xi) \delta^{n}(a+m \delta<\xi<a+(m+n) \delta)$ by a familiar result in the theory of finite differences. It follows that

$$
(-1)^{n} \Delta^{n} f(a+m \delta) \geqq 0 \text {. }
$$

3. Hankel's determinants whose elements are the terms of a completely monotonic sequence. First we introduce the abbreviation

$$
[f(a), f(a+\delta), \cdots, f(a+2 m \delta)]
$$

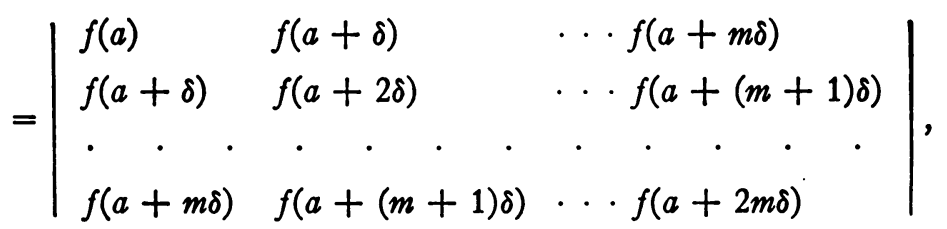

and then prove 
THEOREM 2. If $f(x)$ is completely monotonic in the interval $c<x<\infty$ and if $\delta$ is any positive constant, then the Hankel determinants

$$
[f(a), f(a+\delta), \cdots, f(a+2 m \delta)] \quad(c<a<\infty ; m=0,1,2, \cdots)
$$

are all positive or zero.

Since the sequence

$$
f(a), f(a+\delta), f(a+2 \delta), \cdots
$$

is completely monotonic, there exists* a function $\chi(x)$ bounded and nondecreasing in the interval $0 \leqq x \leqq 1$ such that

$$
f(a+n \delta)=\int_{0}^{1} x^{n} d \chi(x) \quad(n=0,1,2, \cdots) .
$$

Construct the quadratic form

$$
\begin{aligned}
\sum_{i=0}^{n} \sum_{j=0}^{n} f(a & +(i+j) \delta) x_{i} x_{j} \\
& =\int_{0}^{1} \sum_{i=0}^{n} \sum_{j=0}^{n} x^{i+j} x_{i} x_{j} d \chi(x) \\
& =\int_{0}^{1}\left(\sum_{i=0}^{n} x^{i} x_{i}\right)^{2} d \chi(x) \geqq 0 .
\end{aligned}
$$

That this form is never negative follows since $\chi(x)$ is non-decreasing and since the integrand is non-negative. It is known that this implies that the determinants (3.1) are non-negative for $m=0,1,2, \cdots, n$.

THEOREM 3. If $f(x)$ is completely monotonic in the interval $c<x<\infty$, then the determinants

$$
\left[f(a), f^{\prime}(a), \cdots, f^{(2 m)}(a)\right] \quad(c<a<\infty ; m=0,1,2, \cdots)
$$

are positive or zero.

It is a familiar fact that the determinant (3.1) may be written as

$$
\left[f(a), \Delta f(a), \Delta^{2} f(a), \cdots, \Delta^{2 m} f(a)\right] \text {. }
$$

Divide this determinant by $\delta^{m(m+1)}$. By Theorem 2 the quotient is non-negative for all positive $\delta$. Let $\delta$ approach zero. The limit, which is the determinant (3.2), must also be positive or zero.

Clearly the result also holds if the constant $c$ is replaced by a constant $b$

* F. Hausdorff, loc. cit., p. 226. 
greater than $c$, since if $f(x)$ is completely monotonic in $c<x<\infty$ it is also completely monotonic in $b<x<\infty$.

4. Consequences of the vanishing of certain Hankel determinants. We begin by stating two Lemmas, the proofs of which may easily be supplied.

LEMMA 1. If the quadratic form

$$
\sum_{i=0}^{n} \sum_{j=0}^{n} a_{i j} x_{i} x_{j}, \quad a_{i j}=a_{j i}
$$

is non-negative for $x_{n}=1$ and for all values of the other variables, $x_{0}, x_{1}, \cdots$, $x_{n-1}$, then it is non-negative for all values of the variables $x_{0}, x_{1}, \cdots, x_{n}$.

LEMMA 2. If the quadratic form

$$
\sum_{i=0}^{n} \sum_{j=0}^{n} a_{i j} x_{i} x_{j}, a_{i j}=a_{j i},
$$

is non-negative for all values of the variables $x_{0}, x_{1}, \cdots, x_{n}$, and if $a_{00}=0$, then $a_{01}=a_{02}=\cdots=a_{0 n}=0$.

By use of these Lemmas we can prove

THEOREM 4. If $f(x)$ is completely monotonic in the interval $c<x<\infty$, and if

then

$$
\begin{aligned}
{\left[f(a), f^{\prime}(a), \cdots, f^{(2 m)}(a)\right] } & >0 \quad(m=0,1,2, \cdots, k-1), c<a<\infty, \\
& =0 \\
& (m=k),
\end{aligned}
$$

$$
\left[f(a), f^{\prime}(a), \cdots, f^{(2 m)}(a)\right]=0 \quad(m=k, k+1, k+2, \cdots) .
$$

Set $a_{i j}=f^{(i+i)}(a)$. The quadratic form

$$
\sum_{i=0}^{n} \sum_{j=0}^{n} \frac{\Delta^{i+i} f(a)}{\delta^{i+j}} x_{i} x_{j}=\int_{0}^{1}\left(\sum_{i=0}^{n}(x-1)^{i} \frac{x_{i}}{\delta^{i}}\right)^{2} d \chi(x)
$$

is obviously non-negative for every positive value of $\delta$. Allowing $\delta$ to approach zero we see that the quadratic form

$$
\sum_{i=0}^{n} \sum_{j=0}^{n} a_{i j} x_{i} x_{j}
$$

is also positive or zero.

Now consider the quadratic form in the variables $x_{0}, x_{1}, x_{2}, \cdots, x_{k-1}$, $z(k-1<n)$ whose determinant is 


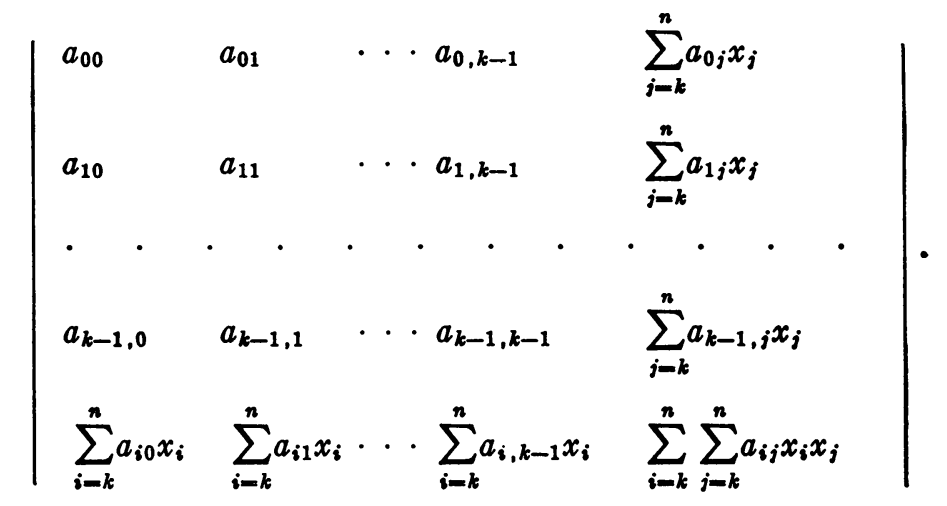

This form reduces to

$$
\sum_{i=0}^{n} \sum_{j=0}^{n} a_{i j} x_{i} x_{j}
$$

for $z=1$, so that it is non-negative, by Lemma 1 , for all values of the variables $x_{0}, x_{1}, \cdots, x_{k-1}, z$. Hence the determinant (4.3) is positive or zero. Expanding it we obtain

$$
\sum_{i=k}^{n} \sum_{j=k}^{n}\left|\begin{array}{ccccc}
a_{00} & a_{01} & \cdots & a_{0, k-1} & a_{0 j} \\
a_{10} & a_{11} & \cdots & a_{1, k-1} & a_{1 j} \\
\cdot & \cdot & \cdot & \cdot & \cdot \\
a_{k-1,0} & a_{k-1,1} & \cdots & a_{k-1, k-1} & a_{k-1, j} \\
a_{i 0} & a_{i 1} & \cdots & a_{i, k-1} & a_{i j}
\end{array}\right| x_{i} x_{j}
$$

This is itself a quadratic form in the variables $x_{k}, x_{k+1}, \cdots, x_{n}$ which is nonnegative. Denote the coefficient of $x_{i} x_{j}$ in this form by $D_{i j}$. Then $D_{k k}=0$ by hypothesis. Consequently, by Lemma 2 ,

$$
D_{k k}=D_{k, k+1}=\cdots=D_{k n}=0
$$

so that

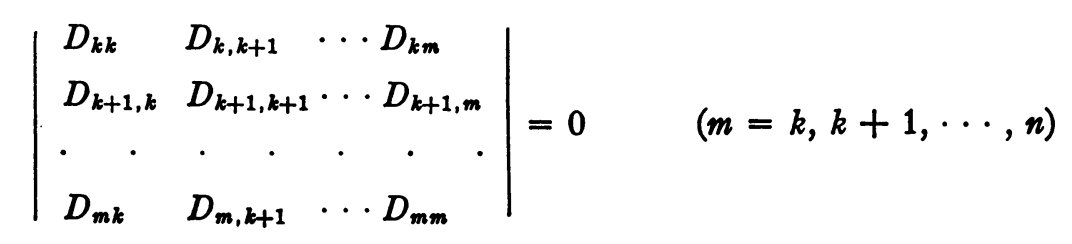

since all the elements of the first row vanish. Now apply Sylvester's determinant theorem* to equation (4.4). It becomes

* See, for example, G. Kowalewski, Einführung in die Determinantentheorie, p. 86. 


$$
\left|\begin{array}{cccc}
a_{00} & a_{01} & \cdots & a_{0, k-1} \\
a_{10} & a_{11} & \cdots & a_{1, k-1} \\
\cdot & \cdot & \cdot & \cdot \\
a_{k-1,0} & a_{k-1,1} & \cdots & a_{k-1, k-1}
\end{array}\right| \cdot\left|\begin{array}{cccc}
a_{00} & a_{01} & \cdots & a_{0 m} \\
a_{10} & a_{11} & \cdots & a_{1 m} \\
\cdot & \cdot & \cdot & \cdot \\
a_{m 0} & a_{m 1} & \cdots & a_{m m}
\end{array}\right|=0 .
$$

Since the first factor is different from zero by hypothesis, it follows that the second factor vanishes for $m=k, k+1, k+2, \cdots, n$. Since $n$ is arbitrary the theorem is proved.

COROllary. If $f(a)=0$, the determinants (4.1) vanish for all $m$.

For, since (4.2) is non-negative, Lemma 1 shows us that $a_{00}=a_{01}=\ldots$ $=a_{0 n}$, from which the result follows at once.

The next result to be proved is

THEOREM 5. Under the conditions of Theorem $4, f(x)$ satisfies a linear differential equation of order $k$ with constant coefficients.

If $k=0$, then by the Corollary to Theorem 4 we have $f(a)=f^{\prime}(a)=f^{\prime \prime}(a)$ $=\cdots=0$. Since every completely monotonic function is analytic, ${ }^{*}$ it follows that $f(x) \equiv 0$. If $k>0$, we have seen that

$$
D_{k m}=0 \quad(m=k, k+1, \cdots) .
$$

This shows that the rank of the matrix

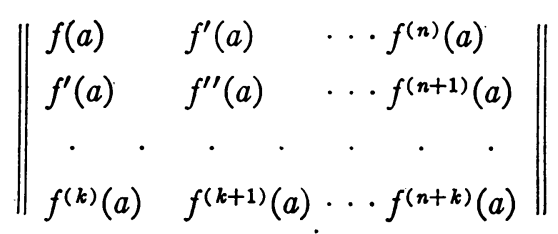

is $k$ for every value of $n \geqq k . \dagger$

Hence there exist constants $K_{0}, K_{1}, \cdots, K_{k}$, not all zero, such that

$$
K_{0} f^{(m)}(a)+K_{1} f^{(m+1)}(a)+\cdots+K_{k} f^{(m+k)}(a)=0 \quad(m=0,1,2, \cdots) .
$$

That is, the analytic function

$$
K_{0} f(x)+K_{1} f^{\prime}(x)+\cdots+K_{k} f^{(k)}(x)
$$

vanishes with all its derivatives at $x=a$, and is consequently identically zero. It remains only to show that $K_{k} \neq 0$. This follows from the hypothesis that (4.1) is different from zero when $m=k-1$. We observe that the differential equation which $f(x)$ satisfies may be put in the form

* Serge Bernstein, Sur la définition et les propriétés des fonctions analytiques d'une variable réelle, Mathematische Annalen, vol. 75 (1914), p. 449.

† G. Kowalewski, loc. cit., p. 53, Theorem 18. 


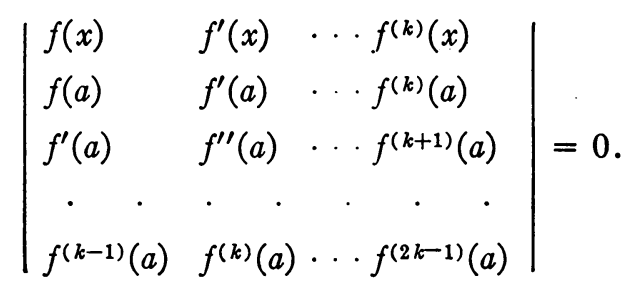

THEOREM 6. Under the conditions of Theorem 4,

$$
(-1)^{m}\left[f^{\prime}(a), f^{\prime \prime}(a), \cdots, f^{(2 m-1)}(a)\right]>0 \quad(m=1,2,3, \cdots, k-1) .
$$

Since the function $-f^{\prime}(x)$ is itself a completely monotonic function, the determinant (4.6) is clearly non-negative for all positive integers $m$ by Theorem 3. Moreover, if (4.6) vanished for $m<k-1$, it would also vanish for $m=k-1$ by Theorem 4 . Consequently, we have only to show that (4.6) is not zero for $m=k-1$. If it were zero, we should have, as in Theorem 5 , that the rank of the matrix

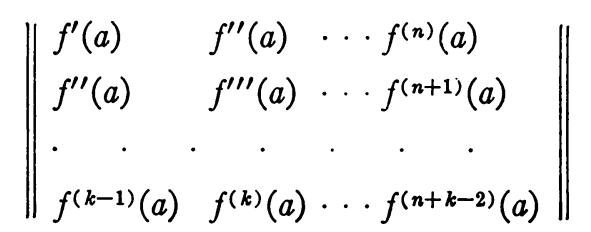

would be at most $k-2$. That is, the determinant (4.1) would vanish for $m=k-1$ contrary to assumption. That (4.6) may vanish for $m=k$ is seen by taking $f(x)=1+e^{-x}, a=0, k=2$. That it need not vanish may be seen by taking $f(x)=e^{-x}+e^{-2 x}, a=0, k=2$.

THEOREM 7. Under the conditions of Theorem 4

$$
\begin{aligned}
& f(x)=c_{1} e^{-\lambda_{1} x}+c_{2} e^{-\lambda_{2} x}+\cdots+c_{k} e^{-\lambda_{k} x}, \\
& 0 \leqq \lambda_{1}<\lambda_{2}<\cdots<\lambda_{k}, c_{i}>0
\end{aligned} \quad(i=1,2, \cdots, k) .
$$

To prove that $f(x)$ has the form (4.7) we must show that the roots of the algebraic equation

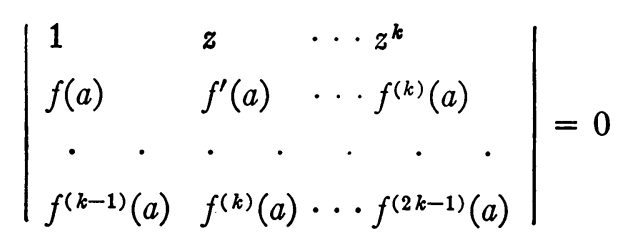

associated with (4.5) are real, distinct and non-negative. To do this we appeal to the theory of continued fractions. If (4.6) is different from zero for $m=k$, then the left-hand side of (4.8) divided by the determinant (4.6) for $m=k$ is, 
except for sign, the denominator of the reduced form of the continued fraction

$$
\begin{aligned}
& \frac{1}{a_{1} z+\frac{1}{a_{2}+1}} \\
& \overline{a_{3} z+1} \\
& +\frac{1}{a_{2 k-1} z+\frac{1}{a_{2 k}}}
\end{aligned}
$$

where

$$
\begin{gathered}
A_{0}=1, B_{0}=1, A_{n}=\left[f(a), f^{\prime}(a), \cdots, f^{(2 n-2)}(a)\right] \\
B_{n}=(-1)^{n}\left[f^{\prime}(a), f^{\prime \prime}(a), \cdots, f^{(2 n-1)}(a)\right], \quad a_{2 n}=\frac{A_{n}^{2}}{B_{n} B_{n-1}}, \quad a_{2 n+1}=\frac{B_{n}^{2}}{A_{n} A_{n+1}} .
\end{gathered}
$$

The rational function of $z$ which this continued fraction represents is defined in the neighborhood of infinity by the series

$$
u(z)=\frac{f(a)}{z}+\frac{f^{\prime}(a)}{z^{2}}+\frac{f^{\prime \prime}(a)}{z^{3}}+\cdots .
$$

By Theorem 6 we see that the $B_{n}$ are all positive, and the $A_{n}$ are all positive by hypothesis, so that the $a_{n}$ are all positive. Under these conditions the roots of (4.8) are known to be distinct and positive.* The left-hand side of (4.8) is the function $Q_{2 n}(z)$ of Stieltjes defined on page 426 of the article cited.

If (4.6) is zero for $m=k$, then the continued fraction development of $u(z)$ is the same as above except that it stops with the term $a_{2 k-1} z$. The denominator of the expanded form is now

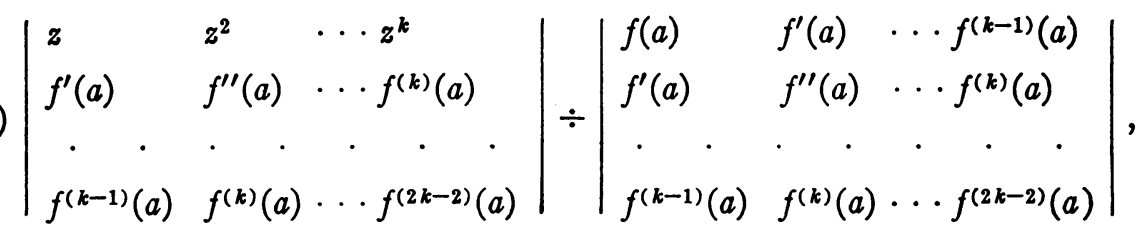

and this is also known to have distinct zeros which are all positive except one which is zero. $\dagger$ It is not difficult to identify the zeros of this function with the roots of equation (4.8). For, since we are assuming that (4.6) vanishes for

* T. J. Stieltjes, Collected Works, p. 411, and p. 426.

$\dagger$ T. J. Stieltjes, loc. cit., p. 411, and p. 427. 
$m=k$ and not for $m=k-1$, we know that constants $L_{1}, L_{2}, \cdots, L_{k-1}$ exist such that

$$
\begin{aligned}
& L_{1} f^{\prime}(a)+L_{2} f^{\prime \prime}(a)+\cdots+L_{k-1} f^{(k-1)}(a)+f^{(k)}(a)=0, \\
& L_{1} f^{\prime \prime}(a)+L_{2} f^{\prime \prime \prime}(a)+\cdots+L_{k} f^{(k)}(a)+f^{(k+1)}(a)=0, \\
& \cdot \cdot \cdot \cdot \cdot \cdot \cdot \cdot \cdot \cdot \cdot \cdot \cdot \cdot \cdot \cdot \cdot \cdot \cdot \cdot \cdot \cdot \cdot L_{k} f^{(2 k-2)}(a)+f^{(2 k-1)}(a)=0 .
\end{aligned}
$$

Expanding the determinant (4.8) according to the elements of the first column we have

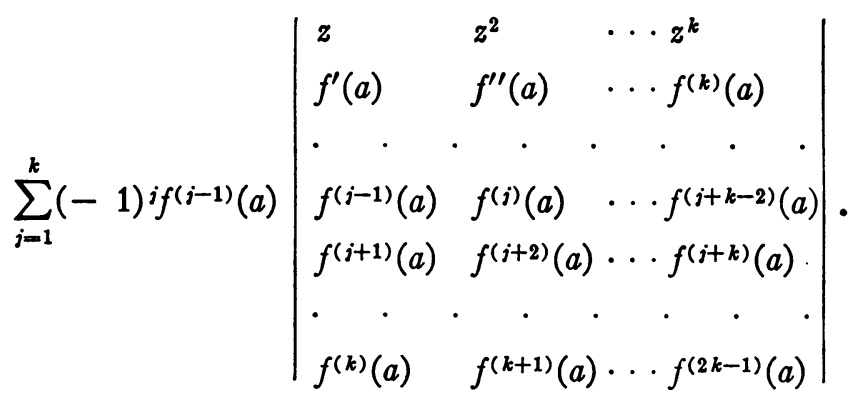

Substitute the values of $f^{(k)}(a), f^{(k+1)}(a), \cdots, f^{(2 k-1)}(a)$, obtained from equations (4.10), in the last row of this determinant. Equation (4.8) thus becomes

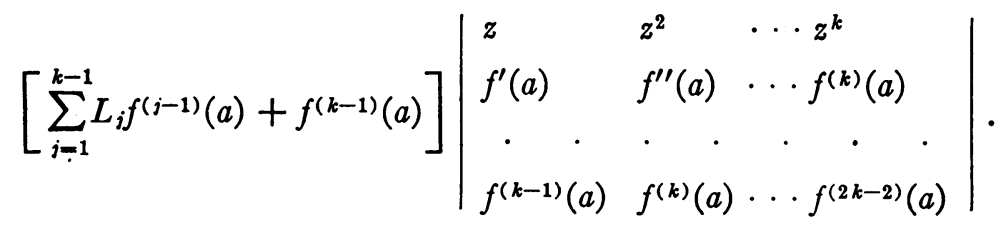

The first factor can not vanish for its vanishing (together with equations (4.10)) would imply that (4.1) would vanish for $m=k-1$ contrary to assumption. Hence the roots of (4.9) are the same as those of (4.11).

It remains only to show that $c_{i}>0$. We have seen that

$$
f(x)=\int_{0}^{\infty} e^{-x t} d \alpha(t)
$$

where $\alpha(t)$ is a step-function with a finite number of jumps. We wish to show that these jumps are all positive. Let $R$ be a number so large that the points of discontinuity of $\alpha(t)$ are all to the left of the point $t=R$. Then if $z$ is a fixed number for which $|z|>R$ the series

$$
\frac{e^{-a t}}{z+t}=e^{-a t}\left[\frac{1}{z}-\frac{t}{z^{2}}+\frac{t^{2}}{z^{3}}-\cdots\right]
$$


is uniformly convergent in the interval $0 \leqq t \leqq R$, so that we have

$$
\begin{aligned}
\int_{0}^{\infty} \frac{e^{-a t}}{z+t} d \alpha(t) & =\frac{1}{z} \int_{0}^{\infty} e^{-a t} d \alpha(t)-\frac{1}{z^{2}} \int_{0}^{\infty} e^{-a t} t d \alpha(t)+\frac{1}{z^{3}} \int_{0}^{\infty} e^{-a t} t^{2} d \alpha(t)-\cdots \\
& =\frac{f(a)}{z}+\frac{f^{\prime}(a)}{z^{2}}+\frac{f^{\prime \prime}(a)}{z^{3}}+\cdots=u(z)
\end{aligned}
$$

We thus have the partial fraction development of $u(z)$ :

$$
u(z)=\frac{c_{1} e^{-a x_{1}}}{z+x_{1}}+\frac{c_{2} e^{-a x_{2}}}{z+x_{2}}+\cdots+\frac{c_{k} e^{-a x_{k}}}{z+x_{k}} .
$$

But the coefficients of this development are known to be positive, ${ }^{*}$ so that the $c_{i}$ are all positive. The theorem is thus completely established.

5. The function $\alpha(t)$ a monotonic function. We are now in a position to prove

THEOREM 8. A necessary and sufficient condition that $f(x)$ should be completely monotonic in the interval $c<x<\infty$ is that

$$
f(x)=\int_{0}^{\infty} e^{-x t} d \alpha(t),
$$

where $\alpha(t)$ is a non-decreasing function of such a nature that the integral converges for $x>c$.

The sufficiency of the condition is obvious since $\dagger$

$$
f^{(n)}(x)=(-1)^{n} \int_{0}^{\infty} e^{-x t} t^{n} d \alpha(t), \quad x>c \quad(n=0,1,2, \cdots) .
$$

To prove the necessity of the condition we appeal to Theorem 7 and to a result of $H$. Hamburger. $\ddagger$ If one of the determinants (4.1) is zero, then $f(x)$ has the form (5.1), $\alpha(t)$ being a step-function with a finite number of positive jumps. If none of these determinants vanishes, then the determinants (4.6) are positive for all $m$, and we are in a position to apply Hamburger's Theorem. $\$$ The function $f(x)$ is thus seen to have the integral expression (5.1).

We note that if $\alpha(t)$ is to be a non-increasing function it is necessary and sufficient that $-f(x)$ should be completely monotonic.

* T. J. Stieltjes, loc. cit., p. 413.

$\dagger$ D. V. Widder, loc. cit., p. 702.

$\ddagger$ H. Hamburger, Bemerkungen zu einer Fragestellung des Berrn Polya, Mathematische Zeitschrift, vol. 7 (1920), p. 304.

\& We must actually apply the theorem to $f(-x)$, but the modifications necessary are obvious. 
6. The function $\alpha(t)$ of bounded variation. We prove the following theorem:

ThEOREM 9. A necessary and sufficient condition that $f(x)$ can be expressed as

$$
f(x)=\int_{0}^{\infty} e^{-x t} d \alpha(t)
$$

with $\alpha(t)$ of bounded variation in every finite interval and the integral absolutely convergent for $x>c$, is that $f(x)$ should be the difference of two functions that are completely monotonic in the interval $c<x<\infty$.

We prove first the necessity of the condition. Suppose $f(x)$ has the form (6.1). We may suppose without loss of generality that $\alpha(0)=0$. Since $\alpha(t)$ is of bounded variation in the interval $0 \leqq t \leqq R$ there exist two non-decreasing functions $P(t)$ and $N(t)$ such that

$$
\begin{aligned}
& \alpha(R)=P(R)-N(R), \\
& u(R)=P(R)+N(R) .
\end{aligned}
$$

Here $u(R)$ is the total variation of $\alpha(t)$ in the interval $0 \leqq t \leqq R$. In this way we see that

$$
f(x)=\lim _{R=\infty}\left[\int_{0}^{R} e^{-x t} d P(t)-\int_{0}^{R} e^{-x t} d N(t)\right]
$$

Since the integral (6.1) is known to converge absolutely, the limit

$$
\int_{0}^{\infty} e^{-x t} d u(t)=\lim _{R=\infty}\left[\int_{0}^{R} e^{-x t} d P(t)+\int_{0}^{R} e^{-x t} d N(t)\right]
$$

exists, so that the integrals

$$
\int_{0}^{\infty} e^{-x t} d P(t), \int_{0}^{\infty} e^{-x t} d N(t)
$$

converge for $x>c$. Hence

$$
f(x)=\int_{0}^{\infty} e^{-x t} d P(t)-\int_{0}^{\infty} e^{-x t} d N(t) .
$$

An application of Theorem 8 now establishes the necessity of the condition.

We turn now to the sufficiency. First suppose that $c \geqq 0$. By virtue of Theorem 8

$$
f(x)=\int_{0}^{\infty} e^{-x t} d P(t)-\int_{0}^{\infty} e^{-x t} d N(t)
$$


where $P(t)$ and $N(t)$ are non-decreasing functions, vanishing at the origin, of such a nature that the integrals converge for $x>c$. For any such value of $x$, constants $K$ and $\epsilon$ exist such that*

$$
P(t)<K e^{t(x-\epsilon)}, \quad N(t)<K e^{t(x-\epsilon)} \quad(0 \leqq t<\infty ; c<x-\epsilon<x) .
$$

Consequently

$$
\begin{aligned}
& \int_{0}^{\infty} e^{-x t} d P(t)=x \int_{0}^{\infty} e^{-x t} P(t) d t, \\
& \int_{0}^{\infty} e^{-x t} d N(t)=x \int_{0}^{\infty} e^{-x t} N(t) d t,
\end{aligned}
$$

the integrals on the right-hand side converging for $x>c$. Now if $\alpha(t)=P(t)$ $-N(t)$, the integral $\int_{0}^{\infty} e^{-x t} d \alpha(t)$ converges absolutely if $x>c$. For, the total variation $u(t)$ of $\alpha(t)$ clearly satisfies the inequality

$$
u(t) \leqq P(t)+N(t) .
$$

This inequality shows that the integral $\int_{0}^{\infty} e^{-x t} u(t) d t$ converges, and hence that

$$
\int_{0}^{\infty} e^{-x t} d u(t)=\lim _{R=\infty} u(R) e^{-x R}+x \int_{0}^{\infty} e^{-x t} u(t) d t .
$$

By virtue of the inequalities (6.2) and (6.3) we see that the indicated limit in (6.4) exists and is zero, so that (6.1) converges absolutely for $x>c$.

The case in which $c<0$ may be reduced to the case just treated by the change of variable $x-c=y$.

We shall next seek to determine a more convenient condition to replace that of Theorem 9. First we shall obtain certain necessary conditions.

TheOREM 10. If the integral

$$
f(x)=\int_{0}^{\infty} e^{-x t} d \alpha(t), \quad \alpha(0)=0,
$$

converges absolutely for $x>c$, then

$$
\begin{array}{ll}
\lim _{x=\infty} f(x)=\alpha(0+), & \\
\lim _{x=\infty} f^{(k)}(x)=0 & (k=1,2,3, \cdots) .
\end{array}
$$

In consideration of Theorem 9 it is sufficient to suppose that $f(x)$ is completely monotonic for $x>c$. Since $f(x)$ is a positive decreasing function it

* D. V. Widder, loc. cit., p. 703, Lemma 2. 
tends to a limit as $x$ becomes infinite. The same is true of every derivative of $f(x)$ since $(-1)^{k} f^{(k)}(x)$ is itself a completely monotonic function. But the limit is zero if $k \geqq 1$. For, suppose

$$
\lim _{x=\infty}(-1)^{k} f^{(k)}(x)=B>0 .
$$

Then

whence

$$
\int_{c+\delta}^{R}(-1)^{k} f^{(k)}(x) d x>B(R-c-\delta), \quad \delta>0
$$

$$
\lim _{R=\infty}(-1)^{k}\left[f^{(k-1)}(R)-f^{(k-1)}(c+\delta)\right]=\infty,
$$

contrary to the fact just proved that $f^{(k-1)}(x)$ approaches a finite limit as $x$ becomes infinite.

If we define a function $\beta(t)$ by the equations

$$
\begin{aligned}
\beta(0) & =0, \\
\beta(t) & =\alpha(0+),
\end{aligned}
$$$$
t>0
$$

it remains only to show that

$$
\lim _{x=\infty} \int_{0}^{\infty} e^{-x t} d[\alpha(t)-\beta(t)]=0 .
$$

The positive function $\gamma(t)=\alpha(t)-\beta(t)$ is continuous at $t=0$. If $\epsilon$ is an arbitrary positive constant, we can find a number $\delta$ so small that

$$
\int_{0}^{\delta} e^{-x t} d \gamma(t)<\gamma(\delta)<\epsilon / 2, \quad x \geqq 0 .
$$

Then we can choose $x$ so large that

$$
\int_{\delta}^{\infty} e^{-x t} d \gamma(t)=e^{-x \delta} \int_{0}^{\infty} e^{-x t} d \gamma(t+\delta)<\epsilon / 2
$$

The latter choice is clearly possible since the integral involving $\gamma(t+\delta)$ approaches a finite limit, ${ }^{*}$ and $e^{-x \delta}$ approaches 0 as $x$ becomes infinite.

THEOREM 11. Under the conditions of Theorem 10 , there exists a constant $M_{8}$ independent of $x$ and of $n$, but dependent on $\delta$, such that

$$
\int_{c+\delta}^{x} \frac{(t-c-\delta)^{n}}{n !}\left|f^{(n+1)}(t)\right| d t<M_{\delta} \quad(\delta>0 ; x \geqq c+\delta, n=0,1,2, \cdots) .
$$

\footnotetext{
* Since $\gamma(t+\delta)$ is monotonic, this follows from the first part of the proof of the present theorem.
} 
By Theorem 9 we have

$$
f(x)=\int_{0}^{\infty} e^{-x t} d \alpha(t)=\int_{0}^{\infty} e^{-x t} d P(t)-\int_{0}^{\infty} e^{-x t} d N(t)
$$

whence

$$
\begin{gathered}
f^{(n+1)}(x)=(-1)^{n+1} \int_{0}^{\infty} e^{-x t} t^{n+1} d P(t)-(-1)^{n+1} \int_{0}^{\infty} e^{-x t} t^{n+1} d N(t) \\
\left|f^{(n+1)}(x)\right| \leqq \int_{0}^{\infty} e^{-x t} t^{n+1} d \gamma(t)
\end{gathered}
$$

where

$$
\gamma(t)=P(t)+N(t)
$$

Then

$$
\int_{c+\delta}^{x} \frac{(y-c-\delta)^{n}}{n !}\left|f^{(n+1)}(y)\right| d y \leqq \int_{0}^{\infty} t^{n+1} d \gamma(t) \int_{c+\delta}^{x} e^{-y t} \frac{(y-c-\delta)^{n}}{n !} d y .
$$

The interchange of the order of integration which we have effected here is permissible since the integral $\int_{0}^{\infty} e^{-x t} t^{n+1} d \gamma(t)$ is uniformly convergent* in the interval $c+\delta \leqq x<\infty$.

The inequality is only strengthened if we replace the upper limit $x$ of the last integral in the above inequality by $\infty$. Thus

$$
\begin{aligned}
\int_{c+\delta}^{x} \frac{(t-c-\delta)^{n}}{n !}\left|f^{(n+1)}(t)\right| d t<\int_{0}^{\infty} t^{n+1} d \gamma(t) \int_{c+\delta}^{\infty} \frac{e^{-x t}(x-c-\delta)^{n}}{n !} d x \\
=\int_{0}^{\infty} t^{n+1} d \gamma(t) e^{-t(c+\delta)} \int_{0}^{\infty} \frac{e^{-x t} x^{n}}{n !} d x=\int_{0}^{\infty} e^{-t(c+\delta)} d \gamma(t)=M_{\delta} .
\end{aligned}
$$

This completes the proof.

We shall now show that the necessary condition established in Theorem 11 is also sufficient, and thus prove

ThEOREM 12. A necessary and sufficient condition that $f(x)$ can be expressed as

$$
f(x)=\int_{0}^{\infty} e^{-x t} d \alpha(t)
$$

with the integral absolutely convergent for $x>c$ and $\alpha(t)$ of bounded variation in every finite interval is that

* D. V. Widder, loc. cit., p. 701. 
(a) $f(x)$ has derivatives of all orders for $x>c$,

(b) a constant $M_{\delta}$ exists independent of $x$ and of $n$ but dependent on $\delta$ such that

$$
\int_{c+\delta}^{x} \frac{(t-c-\delta)^{n}}{n !}\left|f^{(n+1)}(t)\right| d t<M_{\delta} \quad(\delta>0 ; x \geqq c+\delta ; n=0,1,2, \cdots) .
$$

In order to prove this theorem we shall make use of three lemmas.

LEMMA 1. If the functions $\phi_{0}(x), \phi_{1}(x), \phi_{2}(x), \cdots$ are continuous with their first derivatives for $x \geqq x_{0}$ and if a constant $K$ exists such that

$$
\begin{aligned}
\phi_{n}(x) & \leqq \phi_{n+1}(x) \leqq K \\
\phi_{n+1}^{\prime}(x) & \leqq \phi_{n}^{\prime}(x),
\end{aligned} \quad\left(x \geqq x_{0} ; n=0,1,2, \cdots\right),
$$

then the given sequence converges uniformly for $x \geqq x_{0}$.

By hypothesis

$$
\phi_{n+p}^{\prime}(x) \leqq \phi_{n}^{\prime}(x) \quad(p=1,2,3, \cdots)
$$

so that

$$
\begin{aligned}
\int_{x_{0}}^{x} \phi_{n+p}^{\prime}(t) d t & \leqq \int_{x_{0}}^{x} \phi_{n}^{\prime}(t) d t \\
\phi_{n+p}(x)-\phi_{n+p}\left(x_{0}\right) & \leqq \phi_{n}(x)-\phi_{n}\left(x_{0}\right), \\
\phi_{n+p}(x)-\phi_{n}(x) & \leqq \phi_{n+p}\left(x_{0}\right)-\phi_{n}\left(x_{0}\right) .
\end{aligned}
$$

But the sequence $\phi_{0}(x), \phi_{1}(x), \phi_{2}(x), \ldots$ clearly converges for each $x$ since it is an increasing bounded sequence. Hence to an arbitrary positive $\epsilon$ there corresponds an integer $m$ independent of $x$ for $x \geqq x_{0}$ such that

$$
\phi_{n+p}(x)-\phi_{n}(x) \leqq \epsilon \quad(p=0,1,2, \cdots)
$$

when $n>m$. This proves the lemma.

LEMMA 2. If the function $\psi(x)$ is continuous with its first $(k+1)$ derivatives for $x>c$, and if

$$
(-1)^{n} \psi^{(n)}(x) \geqq 0 \quad(x>c ; n=0,1,2, \cdots, k+1),
$$

then

$$
\left|\psi^{(k)}(x)\right| \leqq \frac{\psi(c+\delta) k !}{(x-c-\delta)^{k}} \quad(\delta>0, x>c+\delta) .
$$

By Taylor's theorem we have 


$$
\begin{aligned}
\psi(c+\delta)=\psi(x)+\psi^{\prime}(x)(c+\delta & -x)+\cdots+\psi^{(k)}(x) \frac{(c+\delta-x)^{k}}{k !} \\
& +\int_{x}^{c+\delta} \frac{(c+\delta-t)^{k}}{k !} \psi^{(k+1)}(t) d t \quad(x \geqq c+\delta) .
\end{aligned}
$$

Every term on the right-hand side is positive or zero so that

$$
\psi^{(k)}(x)(c+\delta-x)^{k} \leqq \psi(c+\delta) k !
$$

from which the desired inequality results immediately.

LEMмa 3. Under the conditions of Theorem 12 a constant $A$ exists such that

$$
\begin{aligned}
& \lim _{x=\infty} f(x)=A, \\
& \lim _{x=\infty} f^{(n)}(x)=0 \quad(n=1,2,3, \cdots) .
\end{aligned}
$$

For, integration by parts gives the equality

$$
\begin{aligned}
\int_{c+\delta}^{x} \frac{(t-c-\delta)^{n}}{n !} f^{(n+1)}(t) d t & =f^{(n)}(x) \frac{(x-c-\delta)^{n}}{n !} \\
- & \int_{c+\delta}^{x} \frac{(t-c-\delta)^{n-1}}{(n-1) !} f^{(n)}(t) d t \quad(n=1,2,3, \cdots) .
\end{aligned}
$$

Hence

$$
\begin{aligned}
\left|f^{(n)}(x)\right| \frac{(x-c-\delta)^{n}}{n !} \leqq & \int_{c+\delta}^{x} \frac{(t-c-\delta)^{n}}{n !}\left|f^{(n+1)}(t)\right| d t \\
& \quad+\int_{c+\delta}^{x} \frac{(t-c-\delta)^{n-1}}{(n-1) !}\left|f^{(n)}(t)\right| d t(x \geqq c+\delta),
\end{aligned}
$$

and by condition (b)

$$
\left|f^{(n)}(x)\right| \leqq \frac{2 M_{\delta} n !}{(x-c-\delta)^{n}} \quad(n=1,2,3, \cdots) .
$$

This inequality is sufficient to show that $f^{(n)}(x)$ approaches zero as $x$ becomes infinite $(n=1,2,3, \cdots)$. But

$$
\int_{c+\delta}^{x} f^{\prime}(t) d t=f(x)-f(c+\delta)
$$

Hence if $x$ is allowed to become infinite the function $f(x)$ must approach a limit, since by condition (b) $(n=0)$ the integral

converges absolutely.

$$
\int_{c+8}^{\infty} f^{\prime}(x) d x
$$


We turn now to the proof of the theorem. By Lemma 3 we have

$$
f(x)-A=-\int_{x}^{\infty} f^{\prime}(t) d t
$$

or

$$
|f(x)-A| \leqq \int_{x}^{\infty}\left|f^{\prime}(t)\right| d t
$$

provided that the integral converges. We see that it does converge for $x>c$ by taking $n=0$ in (b). More generally,

$$
\int_{x}^{\infty} \frac{(t-x)^{n-1}}{(n-1) !}\left|f^{(n)}(t)\right| d t \leqq \int_{x}^{\infty} \frac{(t-x)^{n}}{n !}\left|f^{(n+1)}(t)\right| d t .
$$

Both integrals converge for $x>c$ as one sees by again referring to (b). The inequality is established by first noting that

$$
\left|f^{(n)}(x)\right| \leqq \int_{x}^{\infty}\left|f^{(n+1)}(t)\right| d t
$$

and then that

$$
\int_{x}^{\infty} \frac{(t-x)^{n-1}}{(n-1) !}\left|f^{(n)}(t)\right| d t \leqq \int_{x}^{\infty} \frac{(t-x)^{n-1}}{(n-1) !} d t \int_{t}^{\infty}\left|f^{(n+1)}(y)\right| d y .
$$

If we interchange the order of integration on the right-hand side of this inequality, we obtain (6.6). This is permissible since the integrand is positive and since the resulting iterated integral is convergent.* If we set

$$
\phi_{n}(x)=\int_{x}^{\infty} \frac{(t-x)^{n}}{n !}\left|f^{(n+1)}(t)\right| d t,
$$

we may state our result as follows:

$$
|f(x)-A| \leqq \phi_{0}(x) \leqq \phi_{1}(x) \leqq \cdots \leqq \phi_{n}(x) \leqq \cdots \leqq M .
$$

The sequence of functions $\phi_{0}(x), \phi_{1}(x), \phi_{2}(x), \cdots$ has a limit for all $x>c$ which we shall denote by $\phi(x)$. Now the derivative of $\phi_{n}(x)$ is

$$
\phi_{n}^{\prime}(x)=-\int_{x}^{\infty} \frac{(t-x)^{n-1}}{(n-1) !}\left|f^{(n+1)}(t)\right| d t .
$$

To justify the differentiation under the integral sign, set

* E. W. Hobson, The Theory of Functions of a Real Variable and the Theory of Fourier's Series, second edition, vol. 2, p. 346. 


$$
\begin{aligned}
g(x, t) & =\frac{(t-x)^{n}}{n !}\left|f^{(n+1)}(t)\right| & & (t>x), \\
& =0 & & (t \leqq x) .
\end{aligned}
$$

Then

$$
\phi_{n}(x)=\int_{c+\delta}^{\infty} g(x, t) d t .
$$

The functions $g(x, t)$ and $(\partial / \partial x) g(x, t)$ are continuous in the region $x \geqq c+\delta$, $t \geqq c+\delta$ if $n>1$, and the integral

$$
\int_{c+\delta}^{\infty} \frac{\partial g(x, t)}{\partial x} d t
$$

converges uniformly in the interval $x \geqq c+\delta$. For,

$$
\begin{aligned}
\left|\frac{\partial}{\partial x} g(x, t)\right| & \leqq \frac{(t-c-\delta)^{n-1}}{(n-1) !}\left|f^{(n+1)}(t)\right| \quad(x \geqq c+\delta) \\
& \leqq \frac{(t-c-\delta)^{n}}{n !}\left|f^{(n+1)}(t)\right| \quad(t \geqq n+c+\delta) .
\end{aligned}
$$

Since

$$
\int_{c+\delta}^{\infty} \frac{(t-c-\delta)^{n}}{n !}\left|f^{(n+1)}(t)\right| d t
$$

converges, the integral (6.8) is uniformly convergent for $x \geqq c+\delta$. Formula (6.7) also holds if $n=1$, as one may see directly by writing

$$
\phi_{n}(x)=\int_{x}^{\infty} t\left|f^{\prime \prime}(t)\right| d t-x \int_{x}^{\infty}\left|f^{\prime \prime}(t)\right| d t
$$

and differentiating.

In a similar way we have

$$
\phi_{n}^{\prime \prime}(x)=\int_{x}^{\infty} \frac{(t-x)^{n-2}}{(n-2) !}\left|f^{(n+1)}(t)\right| d t \quad(n \geqq 2),
$$

and in general

$$
\phi_{n}^{(k)}(x)=(-1)^{k} \int_{x}^{\infty} \frac{(t-x)^{n-k}}{(n-k) !}\left|f^{(n+1)}(t)\right| d t \quad(k \leqq n) .
$$

It follows that

$$
(-1)^{k} \phi_{n}^{(k)}(x) \geqq 0
$$


Now treating $f^{(m)}(x)$ as we $\operatorname{did} f(x)$, we have

$$
f^{(m)}(x)=-\int_{x}^{\infty} f^{(m+1)}(t) d t \quad(m>0)
$$

$$
\begin{aligned}
\left|f^{(m)}(x)\right| \leqq(-1)^{m} \phi_{m}^{(m)}(x) \leqq(-1)^{m} \phi_{m+1}^{(m)}(x) & \\
& \leqq \cdots \leqq(-1)^{m} \phi_{m+n}^{(m)}(x) \leqq \cdots
\end{aligned}
$$

To show that this sequence has a limit for every $x>c$ we show that it has an upper limit. Let $x_{0}$ be an arbitrary point for which $x_{0}>c+\delta(\delta>0)$. By Lemma 2 we have

$$
\begin{aligned}
\left|\phi_{m+n}^{(m)}(x)\right| & \leqq \frac{\phi(c+\delta) m !}{(x-c-\delta)^{m}} \\
& \leqq \frac{\phi(c+\delta) m !}{\left(x_{0}-c-\delta\right)^{m}} \quad\left(x \geqq x_{0}\right) .
\end{aligned}
$$

The right-hand side of this inequality, being independent of $n$, serves as an upper limit for the sequence (6.9) for all $x \geqq x_{0}$. The functions $\phi_{m+n}^{(m)}(x)$ of the sequence satisfy all the conditions of Lemma 1 at least for $n \geqq 1$ (as we see by replacing $m$ by $m+1$ in the inequalities (6.9)). Consequently, the sequence $\phi_{m}^{(m)}(x), \phi_{m+1}^{(m)}(x), \phi_{m+2}^{(m)}(x), \cdots$ converges uniformly, and its limit is the derivative of the limit of the sequence $\phi_{m}^{(m-1)}(x), \phi_{m+1}^{(m-1)}(x), \phi_{m+2}^{(m-1)}(x), \ldots$ We see in this way that

$$
\lim _{n=\infty} \phi_{m+n}^{(m)}(x)=\phi^{(m)}(x)
$$

and that

$$
\begin{aligned}
|f(x)-A| & \leqq \dot{\phi}(x), \\
\left|f^{(n)}(x)\right| & \leqq(-1)^{n} \phi^{(n)}(x) \quad(n=1,2,3, \cdots) .
\end{aligned}
$$

We are now in a position to show that $f(x)$ is the difference of two completely monotonic functions,

$$
f(x)-A=\frac{f(x)+\phi(x)-A}{2}-\frac{\phi(x)-f(x)+A}{2} .
$$

The inequalities (6.10) lead at once to the following: 


$$
\begin{aligned}
& \frac{f(x)+\phi(x)-A}{2} \geqq 0, \quad \frac{\phi(x)-f(x)+A}{2} \geqq 0, \\
& -\frac{f^{\prime}(x)+\phi^{\prime}(x)}{2} \geqq 0, \quad-\frac{\phi^{\prime}(x)-f^{\prime}(x)}{2} \geqq 0, \\
& (-1)^{n} \frac{f^{(n)}(x)+\phi^{(n)}(x)}{2} \geqq 0,(-1)^{n} \frac{\phi^{(n)}(x)-f^{(n)}(x)}{2} \geqq 0,
\end{aligned}
$$

If $A \geqq 0$ we write

$$
f(x)=\left[A+\frac{f(x)+\phi(x)-A}{2}\right]-\left[\frac{\phi(x)-f(x)+A}{2}\right],
$$

and if $A \leqq 0$,

$$
f(x)=\left[\frac{f(x)+\phi(x)-A}{2}\right]-\left[\frac{\phi(x)-f(x)+A}{2}-A\right] .
$$

In either case $f(x)$ is obviously the difference of two completely monotonic functions. An appeal to Theorem 9 completes the proof of the theorem.

CoRollary. For $f(x)$ to have the form (6.5) it is sufficient that

(a) $f(x)$ should have derivatives of all orders,

( $\left.\mathrm{b}^{\prime}\right) M$ should exist independent of $x$ and of $n$ such that

$$
\left|f^{(n)}(x)\right|<\frac{M \Gamma(n+p)}{(x-c)^{n+p}}(x>c ; n=0,1,2, \cdots)
$$

for some positive constant $p$.

We have only to show that condition $\left(b^{\prime}\right)$ includes condition (b). We can do this as follows:

$$
\begin{gathered}
\int_{c+\delta}^{\infty} \frac{(x-c-\delta)^{n}}{n !}\left|f^{(n+1)}(x)\right| d x=\int_{0}^{\infty} \frac{x^{n}}{n !}\left|f^{(n+1)}(x+c+\delta)\right| d x \\
\leqq \frac{\Gamma(n+p+1)}{n !} M \int_{0}^{\infty} \frac{x^{n}}{(x+\delta)^{n+p+1}} d x=\frac{M \Gamma(p)}{\delta^{p}} .
\end{gathered}
$$

As a simple example take $f(x)=1 / x$. It satisfies the conditions (a) and $\left(b^{\prime}\right)$ for $x>0$, and has the expression 


$$
\frac{1}{x}=\int_{0}^{\infty} e^{-x t} d t
$$

the integral converging for $x>0$.

That conditions (a) and $\left(b^{\prime}\right)$ are not necessary may be seen by noting that the function $f(x)=1$ does not satisfy them. Yet it may be expressed in the form (6.5).

7. The function $\alpha(t)$ an integral. Let us next investigate conditions under which $\alpha(t)$ is an integral, that is, under which $f(x)$ has the form

$$
f(x)=\int_{0}^{\infty} e^{-x t} \phi(t) d t
$$

In this connection we prove

Theorem 13. A necessary and sufficient condition that $f(x)$ can be expressed in the form

$$
f(x)=\int_{0}^{\infty} e^{-x t} \phi(t) d t
$$

with $\phi(t)$ integrable in every finite interval and satisfying an inequality of the form

$$
|\phi(t)| \leqq K e^{c t} \quad(0 \leqq t)
$$

is that

$$
\left|f^{(n)}(x)\right| \leqq \frac{K n !}{(x-c)^{n+1}}(x>c ; n=0,1,2, \cdots) .
$$

Obviously the inequality (7.2) implies the absolute convergence of the integral (7.1) for $x>c$. The necessity of the condition is at once apparent. For, if $x>c$, we have

$$
\begin{aligned}
f^{(n)}(x) & =(-1)^{n} \int_{0}^{\infty} e^{-x t} t^{n} \phi(t) d t \\
\left|f^{(n)}(x)\right| & \leqq \int_{0}^{\infty} e^{-x t} t^{n} K e^{c t} d t=\frac{K n !}{(x-c)^{n+1}} \quad(n=0,1,2, \cdots) .
\end{aligned}
$$

Conversely if (7.3) is satisfied, then

$$
-\frac{K n !}{(x-c)^{n+1}} \leqq(-1)^{n} f^{(n)}(x) \leqq \frac{K n !}{(x-c)^{n+1}},
$$

or 


$$
\begin{gathered}
(-1)^{n}\left[f^{(n)}(x)+(-1)^{n} \frac{K n !}{(x-c)^{n+1}}\right] \geqq 0, \\
(-1)^{n}\left[-f^{(n)}(x)+(-1)^{n} \frac{K n !}{(x-c)^{n+1}}\right] \geqq 0 .
\end{gathered}
$$

This shows that the functions $K(x-c)^{-1}+f(x)$ and $K(x-c)^{-1}-f(x)$ are both completely monotonic in the interval $c<x<\infty$. Hence by_Theorem 8, there exists a non-decreasing function $\beta(t)$ such that

$$
f(x)+\frac{K}{x-c}=\int_{0}^{\infty} e^{-x t} d \beta(t),
$$

the integral converging for $x>c$. But

$$
\frac{K}{x-c}=K \int_{0}^{\infty} e^{-x t} d\left(\frac{e^{c t}}{c}\right)
$$

so that

$$
f(x)=\int_{0}^{\infty} e^{-x t} d \alpha(t)
$$

where

$$
\alpha(t)=\beta(t)-K e^{c t} / c .
$$

Since $\alpha(t)$ is the difference of two monotonic functions, it is a function of bounded variation. But

$$
\frac{K}{x-c}-f(x)=\int_{0}^{\infty} e^{-x t} d\left[\frac{K e^{c t}}{c}-\alpha(t)\right] .
$$

In this way we see that the functions $K e^{c t} c^{-1}+\alpha(t)$ and $K e^{c t} c^{-1}-\alpha(t)$ are both increasing functions. From this fact it follows that

$$
-K e^{c(t+\theta \delta)} \leqq \frac{\alpha(t+\delta)-\alpha(t)}{\delta} \leqq K e^{c(t+\theta \delta)} \quad(0<\delta ; 0<\theta<1)
$$

Allowing $\delta$ to approach zero we obtain

$$
-K e^{c t} \leqq D^{+} \alpha(t) \leqq K e^{c t},
$$

where $D^{+} \alpha(t)$ denotes the upper derivative of $\alpha(t)$ on the right. Now $D^{+} \alpha(t)$ is integrable* since $\alpha(t)$ is of bounded variation and $D^{+} \alpha(t)$ is finite in every finite interval. Consequently, we may write

* E. W. Hobson, loc. cit., vol. 1, p. 549. 


$$
\alpha(x)=\int_{0}^{x} \phi(t) d t
$$

where

$$
\phi(t)=D^{+} \alpha(t),|\phi(t)| \leqq K e^{c t} \quad(0 \leqq t) .
$$

This completes the proof of the theorem. We point out that condition (7.3) implies the vanishing of $f(x)$ at infinity. This also follows indirectly from Theorem 10, since $\alpha(t)$, being an integral, is continuous, and $\alpha(0+)=0$. We further call attention to the fact that condition (7.3) implies condition (b) of Theorem 12. That this should be the case is seen by observing that if $f(x)$ has the form (7.1) it also has the form (6.5) with $\alpha(x)$ defined as

$$
\alpha(x)=\int_{0}^{x} \phi(t) d t .
$$

8. Examples. At this point we illustrate Theorem 13 by a few examples.

A. Take $f(x)=1 / x, \alpha(t)=t, \phi(t)=1, c=0, K=1$. Condition (7.3) is clearly satisfied since

$$
\left|f^{(n)}(x)\right|=\frac{n !}{x^{n+1}} \quad(x>0 ; n=0,1,2, \cdots) .
$$

B. Take $f(x)=e^{-x}, \alpha(t)=0(0 \leqq t<1), \alpha(t)=1(t \geqq 1)$. In this case condition (7.3) should not be satisfied since $\alpha(t)$ is not an integral. We have

$$
\begin{aligned}
& \frac{\left|f^{(n)}(x)\right|(x-c)^{n+1}}{n !}=\frac{e^{-x}(x-c)^{n+1}}{n !} \\
& \max \frac{e^{-x}(x-c)^{n+1}}{n !}=\frac{e^{-(n+1)}(n+1)^{n+1}}{n !}
\end{aligned}
$$

This latter quantity becomes infinite with $n$ so that no constant $K$ exists for condition (7.3) no matter how $c$ may be chosen.

C. Take $f(x)=1, \alpha(t)=1(t>0), \alpha(0)=0$. Here again $\alpha(t)$ is not an integral. Condition (7.3) is not satisfied since $(x-c)$ is not bounded.

D. Take $f(x)=e^{-x} / x, \phi(t)=0(t<1), \phi(t)=1(t \geqq 1), \alpha(t)=0(t \leqq 1), \alpha(t)$ $=t-1(t \geqq 1)$. Here condition (7.3) is satisfied with $c=0, K=1$. For

$$
\begin{aligned}
f^{(n)}(x) & =\frac{e^{-x}}{x} \sum_{p=0}^{n} \frac{(-1)^{n-p}}{x^{p}}\left(\begin{array}{l}
n \\
p
\end{array}\right) p ! \\
\frac{f^{(n)}(x) x^{n+1}}{n !} & =\frac{e^{-x}(-1)^{n}}{n !} \sum_{p=0}^{n}(-1)^{p}\left(\begin{array}{l}
n \\
p
\end{array}\right) p !(x)^{n-p}
\end{aligned}
$$




$$
\begin{aligned}
\frac{\left|f^{(n)}(x)\right| x^{n+1}}{n !} & \leqq e^{-x} \sum_{p=0}^{n} \frac{x^{n-p}}{(n-p) !} \\
& \leqq e^{-x} \sum_{p=0}^{\infty} \frac{x^{p}}{p !}=1
\end{aligned}
$$

E. Take $f(x)=1 / x^{2}, \phi(t)=t$. Then

$$
t \leqq \frac{e^{\epsilon t}}{e \epsilon}
$$

for all positive $\epsilon$. For the function $t e^{-\epsilon t}$ attains its maximum value $(e \epsilon)^{-1}$ at $t=1 / \epsilon$. By Theorem 13 it should follow that

$$
\left|f^{(n)}(x)\right| \leqq n !(e \epsilon)^{-1}(x-\epsilon)^{-n-1}
$$

for all integers $n \geqq 0$, all positive numbers $\epsilon$, and all $x>\epsilon$. Now

$$
\left|f^{(n)}(x)\right|=(n+1) ! x^{-n-2},
$$

so that we should have

or

$$
(n+1) x^{-n-2} \leqq(x-\epsilon)^{-n-1}(\epsilon e)^{-1},
$$

$$
(n+1) \epsilon x^{-1}\left(1-\epsilon x^{-1}\right)^{-n-1} \leqq e^{-1} \text {. }
$$

But the function on the left attains its maximum value $(n+1)^{n+2}(n+2)^{-n-2}$ at $x=\epsilon(n+2)$. As $n$ becomes infinite this maximum value increases and approaches $e^{-1}$ as its limit. The above inequality is thus established. This example serves to illustrate the fact that $|\phi(t)|$ may be equal to $K e^{c t}$ at certain points of $(0, \infty)$ and yet $\left|f^{(n)}(x)\right|$ may never be equal to $K n !(x-c)^{-n-1}$ no matter how large $n$ is taken.

9. Application to Dirichlet series. By use of Theorem 13 we are now able to obtain a condition that is both necessary and sufficient for the development of a function $f(x)$ in a convergent Dirichlet series. We restrict ourselves at first to the case in which the series converges for $x>0$.

THEOREM 14. A necessary and sufficient condition that a real function $f(x)$ can be represented in a Dirichlet series convergent for $x>0$ is that a set of real constants $a_{1}, a_{2}, a_{3}, \cdots, \lambda_{1}, \lambda_{2}, \lambda_{3}, \cdots$,

$$
0 \leqq \lambda_{1}<\lambda_{2}<\lambda_{3}<\cdots, \lim _{k=\infty} \lambda_{k}=\infty,
$$

exist of such a nature that to every positive $\epsilon$ and every integer $k$ there corresponds a number $M$ independent of $n$ and of $x$ such that*

* In (9.1) it is to be understood that $k$ shall also take on the value zero, the quantity in the brace then reducing to $f(x)$. 


$$
\begin{aligned}
\left|\frac{d^{n}}{d x^{n}}\left[\left\{f(x)-\sum_{m=1}^{k} a_{m} e^{-\lambda_{m} x}\right\} x^{-1} e^{\lambda_{k+1} x}\right]\right| & \leqq \frac{\mid M n !}{(x-\epsilon)^{n+1}} \\
& (x>\epsilon ; n=0,1,2, \cdots) .
\end{aligned}
$$

We prove first the necessity of the condition. Let $f(x)$ be the sum of the Dirichlet series

$$
f(x)=\sum_{n=1}^{\infty} a_{n} e^{-\lambda_{n} x}
$$

convergent for $x>0$. Then

$$
f(x)=\int_{0}^{\infty} e^{-x t} d \alpha(t)
$$

where

$$
\alpha(t)=\left\{\begin{array}{lr}
0 & \left(0 \leqq t<\lambda_{1}\right), \\
a_{1}+a_{2}+\cdots+a_{k} & \left(\lambda_{k} \leqq t<\lambda_{k+1} ; k=1,2,3, \cdots\right) .
\end{array}\right.
$$

Since the series and integral converge for $x=\epsilon>0$, there exists*a constant $M$ such that

$$
|\alpha(t)| \leqq M e^{e t} \quad(0 \leqq t<\infty) .
$$

Integrating by parts we obtain

$$
f(x)=x \int_{0}^{\infty} \cdot e^{-x t} \alpha(t) d t
$$

the integrated term disappearing by virtue of (9.2). Now since $\alpha(t)$ is constantly zero in the interval $0 \leqq t<\lambda_{1}$, an obvious change of variable gives us

$$
f(x)=x e^{-\lambda_{1} x} \int_{0}^{\infty} e^{-x t} \alpha\left(t+\lambda_{1}\right) d t
$$

where

$$
\left|\alpha\left(t+\lambda_{1}\right)\right| \leqq M e^{e \lambda_{1}} e^{e t}=M^{\prime} e^{e t}
$$

Now applying Theorem 13 we have

$$
\left|\frac{d^{n}}{d x^{n}}\left\{f(x) x^{-1} e^{\lambda_{1} x}\right\}\right| \leqq \frac{M n !}{(x-\epsilon)^{n+1}}(x>\epsilon ; n=0,1,2 \ldots) .
$$

* D. V. Widder, loc. cit., p. 703, Lemma 2. 
This is the first of the conditions (9.1) corresponding to the case $k=0$. Since

$$
f(x)-\sum_{m=1}^{k} a_{m} e^{-\lambda_{m} x}
$$

is itself a Dirichlet series, the first term of which is $a_{k+1} e^{-\lambda_{k+1} x}$, we have only to apply the result just obtained to the new series to obtain (9.1). The proof of the necessity is thus complete.

We turn now to the proof of the sufficiency of the condition. By Theorem 13, we see that condition (9.1) taken for $k=0$ implies the existence of a function $\alpha(t)$ such that

$$
f(x)=x e^{-\lambda_{1} x} \int_{0}^{\infty} e^{-x t} \alpha(t) d t
$$

where

$$
|\alpha(t)| \leqq M e^{e t} \quad(0 \leqq t<\infty) .
$$

By a linear change of variable, equation (9.3) becomes

$$
f(x)=x \int_{\lambda_{1}}^{\infty} e^{-x t} \alpha\left(t-\lambda_{1}\right) d t=x \int_{0}^{\infty} e^{-x t} \beta(t) d t
$$

where

$$
\beta(t)=\left\{\begin{array}{l}
0 \\
\alpha\left(t-\lambda_{1}\right)
\end{array}\right.
$$$$
\left(\lambda_{1} \leqq t<\infty\right) \text {, }
$$

$$
|\beta(t)| \leqq M e^{-\epsilon \lambda_{1}} e^{\epsilon t} \leqq M e^{e t}
$$

We can now show that $\beta(t)$ is a step-function, or differs from such a function at a set of points of measure zero. Again applying Theorem 13, but now using (9.1) for an arbitrary $k$, we see that

$$
f(x)=\sum_{m=1}^{k} a_{m} e^{-\lambda_{m} x}+x e^{-\lambda_{k+1} x} \int_{0}^{\infty} e^{-x t} \alpha_{k}(t) d t .
$$

As before this may be transformed into

$$
\begin{aligned}
f(x) & =\sum_{m=1}^{k} a_{m} e^{-\lambda_{m} x}+x \int_{\lambda_{k+1}}^{\infty} e^{-x t} \alpha_{k}\left(t-\lambda_{k+1}\right) d t \\
& =\sum_{m=1}^{k} a_{m} e^{-\lambda_{m} x}+x \int_{0}^{\infty} e^{-x t} \beta_{k}(t) d t
\end{aligned}
$$

where 


$$
\beta_{k}(t)=\left\{\begin{array}{lr}
0 & \left(0 \leqq t<\lambda_{k+1}\right), \\
\alpha_{k}\left(t-\lambda_{k+1}\right) & \left(\lambda_{k+1} \leqq t<\infty\right) .
\end{array}\right.
$$

Clearly the summation in this expression may be rewritten as follows:

whence

$$
f(x)=\sum_{m=1}^{k} x \int_{\lambda_{m}}^{\infty} e^{-x t} a_{m} d t+x \int_{0}^{\infty} e^{-x t} \beta_{k}(t) d t
$$

$$
\begin{gathered}
f(x)=x \int_{0}^{\infty} e^{-x t} \gamma_{k}(t) d t, \\
\gamma_{k}(t)=\left\{\begin{array}{lr}
0 & \left(0 \leqq t<\lambda_{1}\right), \\
a_{1}+a_{2}+\cdots+a_{\nu} & \left(\lambda, \leqq t<\lambda_{\nu+1} ; \nu=1,2, \cdots, k\right), \\
\beta_{k}(t)+\sum_{m=1}^{k} a_{m} & \left(\lambda_{k+1} \leqq t<\infty\right) .
\end{array}\right.
\end{gathered}
$$

If we now make use of the uniqueness theorem, ${ }^{*}$ we see that $\gamma(t)$ must coincide with $\beta(t)$ almost everywhere. By allowing $k$ to become infinite, we see that $\beta(t)$ differs from a step-function $\gamma(t)$ at most at a set of points of measure zero. Since $\gamma(t)$ is a step-function it follows that the inequality (9.4) implies

$$
|\gamma(t)| \leqq M e^{e t} \quad(0 \leqq t<\infty) .
$$

Hence, on integrating by parts, we obtain

$$
f(x)=\int_{0}^{\infty} e^{-x t} d \gamma(t)=\sum_{n=1}^{\infty} a_{n} e^{-\lambda_{n} x} .
$$

The integral and series converge for $x>\epsilon$ by virtue of (9.5). But $\epsilon$ was an arbitrary positive quantity. The above argument repeated for any positive $\epsilon$ must always lead to the same Dirichlet series since expansion in such a series is unique. It follows that the series converges for $x>0$, and the proof is complete.

We can now see that the restriction of convergence for $x>0$ was not an essential one. For, if the series (9.11) converges for $x>c$, then the series

$$
f(x+c)=\sum_{n=1}^{\infty} a_{n} e^{-\lambda_{n} c} e^{-\lambda_{n} x}
$$

converges for $x>0$, and we can apply Theorem 14. The inequality (9.1) in the statement of that theorem must be replaced by the following one:

$$
\left|\frac{d^{n}}{d x^{n}}\left\{\left[f(x)-\sum_{m=1}^{k} a_{m} e^{-\lambda_{m} x}\right](x-c)^{-1} e^{\lambda_{k+1}} x\right\}\right| \leqq \frac{M n !}{(x-c-\epsilon)^{n+1}} .
$$

* D. V. Widder, loc. cit., p. 705. 
If we restrict ourselves to Dirichlet series with positive coefficients a theorem that is much simpler in statement may be obtained by use of Theorem 9 .

TheOREM 15. A necessary and sufficient condition that $f(x)$ can be represented in a Dirichlet series with positive coefficients convergent for $x>c$ is that a set of constants

$$
\begin{gathered}
a_{1}, a_{2}, a_{3}, \cdots, \lambda_{1}, \lambda_{2}, \lambda_{3}, \cdots, \\
0 \leqq \lambda_{1}<\lambda_{2}<\lambda_{3}<\cdots, \lim _{k=\infty} \lambda_{k}=\infty,
\end{gathered}
$$

exist such that the function

$$
\left(f(x)-\sum_{m=1}^{k} a_{m} e^{-\lambda_{m} x}\right) e^{\lambda_{k+1} x}
$$

is a completely monotonic function in the interval $c<x<\infty$ for $k=0,1,2, \ldots$.

We omit the proof since it follows closely that of Theorem 14, making application of Theorem 9 instead of Theorem 13.

10. Relation between completely monotonic functions and completely monotonic sequences. We turn now to the discussion of the following problem. Given an infinite set of constants $a_{0}, a_{1}, a_{2}, \cdots$. Under what conditions is it possible to determine a completely monotonic function $f(x)$ such that $f(n)=a_{n}$ for $n=0,1,2, \ldots$ ? In order to simplify the statement of the solution of this problem we introduce a

Definition. A completely monotonic set of constants $a_{0}, a_{1}, a_{2}, \cdots$ is minimal if decreasing $a_{0}$ makes of it a set which is no longer completely monotonic.

That there exist completely monotonic sets which are not minimal may be seen by noting that increasing the first element of a set which is completely monotonic leaves it so. By a theorem of Hausdorf* it is known that any completely monotonic set $a_{0}, a_{1}, a_{2}, \cdots$ can be represented in the form

$$
a_{n}=\int_{0}^{1} t^{n} d \phi(t) \quad(n=0,1,2, \cdots)
$$

where $\phi(t)$ is a non-decreasing function. Moreover, the representation in this form is unique if "normalized" functions $\phi(t)$ only are admitted, that is, functions for which

$$
\phi(0)=0, \quad \phi(t)=\frac{\phi(t+0)+\phi(t-0)}{2} \quad(0<t<1) .
$$

We first establish the following

\footnotetext{
* F. Hausdorff, loc. cit., p. 226.
} 
LEMma. The completely monotonic set $a_{0}, a_{1}, a_{2}, \cdots$ is minimal if and only if the function $\phi(t)$ of its Hausdorff representation is continuous at $t=0$.

We prove first the necessity of the condition, showing that if $\phi(0+)>0$ the set is not minimal. Define a function $\psi(t)$ continuous at $t=0$ by the equations

$$
\psi(t)=\left\{\begin{array}{lr}
0 & (t=0), \\
\phi(t)-\phi(0+) & (0<t \leqq 1) .
\end{array}\right.
$$

Then

$$
\begin{aligned}
\int_{0}^{1} t^{n} d \psi(t) & =\int_{0}^{1} t^{n} d \phi(t)=a_{n} \quad(n=1,2,3, \cdots) \\
& =\int_{0}^{1} d \phi(t)-\phi(0+)=a_{0}-\phi(0+)
\end{aligned}
$$

Since $\psi(t)$ is itself a non-decreasing function, the set $a_{0}-\phi(0+), a_{1}, a_{2}, \cdots$, is itself completely monotonic, so that the given set can not have been minimal.

Conversely, if $\phi(0+)=0$, then the set $a_{0}, a_{1}, a_{2}, \cdots$ is minimal. If it were not so, a positive constant $k$ would exist such that $a_{0}-k, a_{1}, a_{2}, \cdots$ would be a completely monotonic set. That is, a unique normalized non-decreasing function $\psi(t)$ would exist satisfying the equations

$$
\begin{aligned}
a_{n} & =\int_{0}^{1} t^{n} d \psi(t) \quad(n=1,2,3, \cdots), \\
a_{0}-k & =\int_{0}^{1} d \psi(t) .
\end{aligned}
$$

But we clearly have

$$
\begin{aligned}
a_{n} & =\int_{0}^{1} t^{n} d \bar{\psi}(t) \quad(n=1,2,3, \cdots), \\
a_{0}-k & =\int_{0}^{1} d \bar{\psi}(t),
\end{aligned}
$$

where

$$
\begin{array}{ll}
\bar{\psi}(t)=\phi(t)-k & (0<t \leqq 1), \\
\bar{\psi}(0) & =0 .
\end{array}
$$

Hence $\bar{\psi}(t) \equiv \psi(t)$, for $\bar{\psi}(t)$ is clearly normalized if $\phi(t)$ is. But $\bar{\psi}(t)$ is not a 
non-decreasing function since $\bar{\psi}(0+)-\bar{\psi}(0)=-k<0$. Since $\psi(t)$ is non-decreasing we have a contradiction, thus completing the proof of the lemma.

By use of this result we are able to prove

THEOREM 16. A necessary and sufficient condition that there should exist a function $f(x)$ completely monotonic in the interval $0 \leqq x<\infty$ such that $f(n)=a_{n}$ for $n=0,1,2, \cdots$ is that the set $a_{0}, a_{1}, a_{2}, \cdots$ should be a minimal completely monotonic set.

We begin with the sufficiency of the condition. Suppose the set $a_{0}, a_{1}$, $a_{2}, \cdots$ to be of specified type. Then

$$
a_{n}=\int_{0}^{1} t^{n} d \phi(t) \quad(n=0,1,2, \cdots),
$$

where $\phi(t)$ is a non-decreasing function vanishing at $t=0$ and continuous there. Since $\phi(t)$ is continuous at $t=0$ we have

$$
a_{n}=\lim _{\epsilon=0} \int_{\epsilon}^{1} t^{n} d \phi(t) \quad(n=0,1,2, \cdots) .
$$

Make the change of variable $t=e^{-\nu}$. Then

$$
a_{n}=\lim _{\epsilon=0} \int_{0}^{-\log e} e^{-n y} d \alpha(y)=\int_{0}^{\infty} e^{-n y} d \alpha(y)
$$

where

$$
\alpha(y)=-\phi\left(e^{-y}\right) .
$$

The function $\alpha(y)$ is clearly non-decreasing. The function

$$
f(x)=\int_{0}^{\infty} e^{-x t} d \alpha(t)
$$

is completely monotonic in the interval $0<x<\infty$ since the integral converges for $x>0$. The function $f(x)$ is continuous* in the interval $0 \leqq x<\infty$ since the integral converges for $x=0$. Since $f(n)=a_{n}$, the proof of the sufficiency of the condition is complete.

Consider now the necessity of the condition. Suppose that a function $f(x)$, completely monotonic in the interval $0 \leqq x<\infty$, exists such that $f(n)=a_{n}$ for $n=0,1,2, \ldots$. We show first that the set of constants $a_{0}, a_{1}, a_{2}, \ldots$ is completely monotonic. The point is not covered by Theorem 1 since it is not known that the point $x=0$ is an interior point of an interval in which $f(x)$ is completely monotonic. By Theorem 8 we have

* D. V. Widder, loc. cit., p. 701. 


$$
f(x)=\int_{0}^{\infty} e^{-x t} d \alpha(t)
$$

where $\alpha(t)$ is a non-decreasing function and the integral converges for $x>0$. We can show that the integral also converges for $x=0$. For suppose that it diverged. Since $\alpha(t)$ is monotonic we should then have $\alpha(\infty)=\infty$. In that case $\lim _{x=0} f(x)=\infty$. For, if $x$ is a fixed positive quantity, we have

$$
\begin{aligned}
f(x) & =x \int_{0}^{\infty} e^{-x t} \alpha(t) d t \\
& =x \int_{0}^{1 / x} e^{-x t} \alpha(t) d t+x \int_{1 / x}^{\infty} e^{-x t} \alpha(t) d t .
\end{aligned}
$$

Since $\alpha(t) \geqq 0$ and $e^{-x t}>0$ we have

whence

$$
x \int_{0}^{1 / x} e^{-x t} \alpha(t) d t \geqq 0,
$$

$$
f(x) \geqq x \int_{1}^{\infty} e^{-x t} \alpha(t) d t .
$$

But $\alpha(t) \geqq \alpha(1 / x)$ in the interval $1 / x \leqq t<\infty$. Hence

Consequently

$$
f(x) \geqq \alpha(1 / x) x \int_{1 / 2}^{\infty} e^{-x t} d t=\alpha(1 / x) / e .
$$

$$
\lim _{x=0} f(x)=\alpha(\infty) / e=\infty .
$$

But since $f(x)$ is assumed continuous at $x=0$,

$$
\lim _{x=0} f(x)=f(0)=a_{0} .
$$

The assumption that the integral (10.1) diverged for $x=0$ was false. The integral thus defined for $x=0$ must be equal to $f(0)=a_{0}$ since both the integral and the function $f(x)$ are continuous at $x=0$. Hence

$$
a_{n}=\int_{0}^{\infty} e^{-n t} d \alpha(t) \quad(n=0,1,2, \cdots) .
$$

It follows that the set $a_{0}, a_{1}, a_{2}, \cdots$ is completely monotonic since

$$
(-1)^{k} \Delta^{k} a_{m}=\int_{0}^{\infty}\left(1-e^{-t}\right)^{k} e^{-m t} d \alpha(t) \geqq 0(k=0,1,2, \cdots ; m=0,1,2, \cdots) .
$$


It remains only to show that this set is minimal. We have

where

$$
a_{n}=\lim _{R=\infty} \int_{0}^{R} e^{-n t} d \alpha(t)=\lim _{R=\infty} \int_{0^{-R}}^{1} t^{n} d \beta(t),
$$

$$
\beta(t)=-\alpha\left(\log \frac{1}{t}\right) \quad(0<t \leqq 1) .
$$

The function $\beta(t)$ is undefined for $t=0$. If $\beta(0)$ is defined as $-\alpha(\infty)$, we have $\beta(0)=\beta(0+)$ and

$$
a_{n}=\int_{0}^{1} t^{n} d \beta(t)
$$

Since $\beta(t)$ is continuous at $t=0$, the set $a_{0}, a_{1}, a_{2}, \cdots$ must be minimal, and the proof is complete.

11. The integral $\int_{0}^{1} e^{x t} d \alpha(t)$. We turn now to the determination of conditions both necessary and sufficient for the representation of a function $f(x)$ in the form

$$
f(x)=\int_{0}^{1} e^{x t} d \alpha(t)
$$

where $\alpha(t)$ is a non-decreasing function. First consider necessary conditions. If $f(x)$ has the above representation it is clearly an entire function. Let $x_{0}$ be any real value. Then

$$
\begin{aligned}
f^{(n)}\left(x_{0}\right) & =\int_{0}^{1} e^{x_{0} t} t^{n} d \alpha(t) \\
& =\int_{0}^{1} t^{n} d \beta(t),
\end{aligned}
$$

where

$$
\begin{array}{ll}
\beta(t) & =\int_{0}^{t} e^{x_{0} y} d \alpha(y) \quad(0<t \leqq 1), \\
\beta(0) & =0 .
\end{array}
$$

Since $\beta(t)$ is a non-decreasing function, the sequence $f\left(x_{0}\right), f^{\prime}\left(x_{0}\right), f^{\prime \prime}\left(x_{0}\right), \ldots$ is completely monotonic. In particular if $\alpha(t)$ is continuous at $t=0, \beta(t)$ is also continuous there, and the above sequence is minimal. We can now show that these necessary conditions are sufficient. Let $f(x)$ be a function with derivatives of all orders at $x=x_{0}$ and such that the sequence of its successive derivatives there is completely monotonic. That is,

$$
f(x)=\sum_{n=0}^{\infty} \frac{f^{(n)}\left(x_{0}\right)\left(x-x_{0}\right)^{n}}{n !} .
$$


This function is entire. For

$$
0 \leqq f^{(n)}\left(x_{0}\right) \leqq f\left(x_{0}\right) \quad(n=0,1,2, \cdots),
$$

and

$$
\begin{aligned}
f(x)=\sum_{n=0}^{\infty} \frac{f^{(n)}\left(x_{0}\right)\left(x-x_{0}\right)^{n}}{n !} \ll f\left(x_{0}\right) \sum_{n=0}^{\infty} \frac{\left|x-x_{0}\right|^{n}}{n !} & =f\left(x_{0}\right) e^{\left|x-x_{0}\right|} \\
& (-\infty<x<\infty) .
\end{aligned}
$$

Now by Hausdorff's theorem, it is possible to determine a non-decreasing bounded function $\beta(t)$ such that

whence

$$
f^{(n)}\left(x_{0}\right)=\int_{0}^{1} t^{n} d \beta(t)
$$

$$
f(x)=\sum_{n=0}^{\infty} \frac{\left(x-x_{0}\right)^{n}}{n !} \int_{0}^{1} t^{n} d \beta(t) .
$$

For any fixed value of $x$ the series

$$
e^{\left(x-x_{0}\right) t}=\sum_{n=0}^{\infty} \frac{\left(x-x_{0}\right)^{n} t^{n}}{n !}
$$

is uniformly convergent in the interval $0 \leqq t \leqq 1$, since

$$
\sum_{n=0}^{\infty} \frac{\left(x-x_{0}\right)^{n} t^{n}}{n !} \ll \sum_{n=0}^{\infty} \frac{\left|x-x_{0}\right|^{n}}{n !}=e^{\left|x-x_{0}\right|} .
$$

Hence it may be integrated term by term with respect to the monotonic function $\beta(t)$. That is,

so that

$$
\int_{0}^{1} e^{\left(x-x_{0}\right) t} d \beta(t)=\sum_{n=0}^{\infty} \frac{\left(x-x_{0}\right)^{n}}{n !} \int_{0}^{1} t^{n} d \beta(t)=f(x),
$$

where

$$
f(x)=\int_{0}^{1} e^{x t} d \alpha(t)
$$

$$
\begin{aligned}
& \alpha(t)=\int_{0}^{t} e^{-x_{0} y} d \beta(y) \quad(0<t \leqq 1), \\
& \alpha(0)=0 .
\end{aligned}
$$

Again we see that $\alpha(t)$ is non-decreasing, is continuous at $t=0$ if $\beta(t)$ is continuous there. We have seen that $\beta(t)$ has this property if and only if the sequence $f\left(x_{0}\right), f^{\prime}\left(x_{0}\right), \cdots$ is minimal. We have thus established 
ThEOREM 17. A necessary and sufficient condition that a function $f(x)$ can be represented in the form

$$
f(x)=\int_{0}^{1} e^{x t} d \alpha(t)
$$

with $\alpha(t)$ a non-decreasing bounded function (continuous at $t=0$ ) is that $f(x)$ should have derivatives of all orders at a point $x=x_{0}$ which form a (minimal) completely monotonic set, $f\left(x_{0}\right), f^{\prime}\left(x_{0}\right), f^{\prime \prime}\left(x_{0}\right), \cdots$.

12. Generalized derivatives. Let us now combine the results of Theorems 16 and 17. If $\alpha(t)$ is non-decreasing and continuous at $t=0$, the set $f\left(x_{0}\right)$, $f^{\prime}\left(x_{0}\right), f^{\prime \prime}\left(x_{0}\right), \cdots$ is a minimal completely monotonic set, so that there exists a function $\phi(x)$ that is completely monotonic in $0 \leqq x<\infty$ such that

$$
\phi(n)=f^{(n)}\left(x_{0}\right) \quad(n=0,1,2, \cdots) .
$$

It is natural to inquire if there is not some sense in which this equation holds for non-integral values of $n$. We shall show that if $f^{(n)}\left(x_{0}\right)$ is replaced by the generalized derivative of Riemann (slightly modified* to meet our need) then the above equation holds for all $n>0$. We define the generalized derivative of positive order $\rho$ of a function $f(x)$ as

$$
{ }_{-\infty} D_{x}^{p} f(x)=\int_{0}^{\infty} \frac{t^{-\nu} f^{(m+1)}(x-t)}{\Gamma(1-\nu)} d t \quad(0 \leqq \nu<1),
$$

where $m+\nu=\rho$ and $m$ is the largest integer contained in $\rho$. Clearly if $\rho$ is an integer $m$ and if $f^{(m)}(x)$ is a function which vanishes for $x=-\infty$, then

$$
{ }_{-\infty} D_{x}^{m} f(x)=f^{(m)}(x) .
$$

With this definition at hand we can now prove

THEOREM 18. The generalized derivative ${ }_{-\infty} D_{x} \rho f(x)$ is a completely monotonic function of $\rho$ in the interval $\rho \geqq 0$ for every $x$ if and only if

$$
f(x)=\int_{0}^{1} e^{x t} d \alpha(t)
$$

where $\alpha(t)$ is a bounded non-decreasing function that is continuous at $t=0$.

To prove the sufficiency of the condition we show first that the integral

* This generalized derivative, ${ }_{-\infty} D_{x}{ }^{\rho} f(x)$, is ordinarily defined for positive values of $\rho$ through the medium of its values for negative $\rho$. For the functions under consideration, however, it need not exist for negative $\rho$. The definition we give is legitimate since for positive integral values of $\rho$ the generalized derivative reduces to the ordinary derivative. 


$$
{ }_{-\infty} D_{x}^{\rho} f(x)=\frac{1}{\Gamma(1-\nu)} \int_{0}^{\infty} t^{-\nu} d t \int_{0}^{1} e^{x y-t y} y^{m+1} d \alpha(y)
$$

converges. The integral is improper both on account of the infinite upper limit of the integral and because the integrand becomes infinite at $t=0 \mathrm{i}$ $\nu \neq 0$. For every $x$ the integral

$$
\int_{0}^{1} e^{y(x-t)} y^{m+1} d \alpha(y)
$$

is a continuous function of $t$, so that the integrand of (12.1) is $O\left(t^{-\nu}\right)$ as $t$ approaches zero. Since $\nu<1$ we are assured of the convergence of the integral if the upper limit $\infty$ is replaced by any positive finite limit. We must now investigate the behavior of the integrand as $t$ becomes infinite. We show that for any fixed $x$ and for $m \geqq 0$

For

$$
\int_{0}^{1} e^{y(x-t)} y^{m+1} d \alpha(y)=o\left(t^{-1}\right) .
$$

$$
\begin{array}{r}
\int_{0}^{1} e^{y(x-t)} y^{m+1} d \alpha(y)=\alpha(1) e^{x-t}-\int_{0}^{1} \alpha(y)\left\{(m+1) y^{m} e^{y(x-t)}\right. \\
\left.+(x-t) e^{y(x-t)} y^{m+1}\right\} d y
\end{array}
$$

The first term on the right-hand side of this equation is clearly $o\left(t^{-1}\right) !$ The integral on the right-hand side may be set equal to $I_{1}+I_{2}$ where

$$
\begin{aligned}
& I_{1}=\int_{0}^{t^{-1 / 2}} \alpha(y)\left\{(m+1) y^{m} e^{y(x-t)}+(x-t) e^{y(x-t)} y^{m+1}\right\} d y, \\
& I_{2}=\int_{t^{-1 / 2}}^{1} \alpha(y)\left\{(m+1) y^{m} e^{y(x-t)}+(x-t) e^{y(x-t)} y^{m+1}\right\} d y .
\end{aligned}
$$

Applying the second law of the mean to $I_{1}$ we obtain

$$
\begin{array}{r}
I_{1}=\alpha\left(t^{-1 / 2}\right) \int_{\xi}^{t^{-1 / 2}}\left\{(m+1) y^{m} e^{y(x-t)}+(x-t) e^{y(x-t)} y^{m+1}\right\} d y \quad\left(0 \leqq \xi<t^{-1 / 2}\right), \\
=\alpha\left(t^{-1 / 2)}\left\{(m+1) \eta^{m} \int_{\xi}^{t^{-1 / 2}} e^{y(x-t)} d y+(x-t) \eta^{m} \int_{\xi}^{t^{-1 / 2}} y e^{\nu(x-t)} d y\right\}\right. \\
\quad\left(\xi<\eta<t^{-1 / 2}\right) .
\end{array}
$$

Hence if $t>x$ we have 


$$
\begin{aligned}
\left|I_{1}\right|<\alpha\left(t^{-1 / 2}\right)(m+1)\left(t^{-m / 2}\right) & \frac{1-e^{x t^{-1 / 2}-t^{1 / 2}}}{t-x} \\
& +\alpha\left(t^{-1 / 2}\right)(t-x) t^{-m / 2}\left\{\frac{1+e^{x t^{-1 / 2}-t^{1 / 2}}\left[1+(t-x) t^{-1 / 2}\right]}{(t-x)^{2}}\right\} .
\end{aligned}
$$

Since $\alpha\left(t^{-1 / 2}\right)$ approaches zero as $t$ becomes infinite, it is clear that $I_{1}=o\left(t^{-1}\right)$ if $m \geqq 0$. For $I_{2}$ we have the following equation and inequalities:

$$
\begin{aligned}
I_{2} & =\alpha(\xi)\left\{(m+1) \xi^{m}+(x-t) \xi^{m+1}\right\} \int_{t^{-1 / 2}}^{1} e^{\nu(x-t)} d t \quad\left(t^{-1 / 2}<\xi<1\right), \\
\left|I_{2}\right| & \leqq \alpha(1)\{(m+1)+(t-x)\} \int_{t^{-1 / 2}}^{1} e^{\nu(x-t)} d t, \\
\left|I_{2}\right| & \leqq \alpha(1)\{(m+1)+(t-x)\}\left\{e^{x-t}-e^{x t^{-1 / 2}-t^{1 / 2}}\right\}(t-x)^{-1},
\end{aligned}
$$

from which we see that $I_{2}$ is also $o(1 / t)$. The integrand of (12.1) when multiplied by $t^{1+\nu}$ approaches zero as $t$ becomes infinite. By the usual limit test for convergence we infer therefore that the integral converges if $\nu>0$. If $\nu=0$ the integral may be integrated in finite form, and we are assured of convergence since

$$
\lim _{t=\infty} f^{(m)}(x-t)=0 \quad(m=0,1,2, \cdots)
$$

by virtue of Theorem 10 .

We show next that it is permissible to interchange the order of integration in (12.1). We rewrite that integral as

$$
\begin{aligned}
{ }_{-\infty} D_{x}^{\rho} f(x)=\frac{1}{\Gamma(1-\nu)} & \int_{0}^{\infty} t^{-\nu} \alpha(1) e^{x-t} d t \\
& +\frac{1}{\Gamma(1-\nu)} \int_{0}^{\infty} t^{-\nu+1} d t \int_{0}^{1} \alpha(y) e^{y(x-t)} y^{m+1} d y \\
& -\frac{x}{\Gamma(1-\nu)} \int_{0}^{\infty} t^{-\nu} d t \int_{0}^{1} \alpha(y) e^{y(x-t)} y^{m+1} d y \\
& -\frac{1}{\Gamma(1-\nu)} \int_{0}^{\infty} t^{-\nu} d t \int_{0}^{1} e^{y(x-t)} y^{m}(m+1) \alpha(y) d y
\end{aligned}
$$

and apply a familiar theorem* to each of the iterated integrals.

We must show

(a) that the two repeated integrals in opposite orders over the domain $(0,0 ; 1, R)$ exist and have equal values for every positive $R$,

* E. W. Hobson, loc. cit., vol. 2, p. 398. 
(b) that the iterated integrals of (12.3) converge,

(c) that the integrands are non-negative.

The last of these conditions is obvious. Since each term on the right-hand side of (12.2) has been shown to be $o\left(t^{-1}\right)$, (b) follows at once. To prove (a) we have only to note again that the integrands are non-negative and apply a known theorem.* Consequently

$$
\begin{gathered}
{ }_{-\infty} D_{x}^{\rho} f(x)=\alpha(1) e^{x}-x \int_{0}^{1} \alpha(y) e^{x y} y^{m+1} y^{y-1} d y+(1-\nu) \int_{0}^{1} \alpha(y) e^{x y} y^{m+1} y^{y-2} d y \\
-\int_{0}^{1} \alpha(y) e^{y x}(m+1) y^{m} y^{y-1} d y,
\end{gathered}
$$

since

$$
\int_{0}^{\infty} t^{-v} e^{-t y} d t=y^{\nu-1} \Gamma(1-\nu) \quad(\nu<1) .
$$

But

Consequently,

$$
\begin{aligned}
\int_{0}^{1} e^{x y} y^{m+\nu} d \alpha(y)=\alpha(1) e^{x}-x \int_{0}^{1} \alpha(y) e^{x y} y^{m+\nu} d y \\
-(m+\nu) \int_{0}^{1} \alpha(y) e^{x y} y^{m+\nu-1} d y .
\end{aligned}
$$

$$
{ }_{-\infty} D_{x}^{\rho} f(x)=\int_{0}^{1} e^{x y} y^{m+v} d \alpha(y)=\int_{0}^{1} e^{x y} y^{\rho} d \alpha(y) \quad(\rho \geqq 0) .
$$

To show that this is a completely monotonic function of $\rho$ set $y=e^{-u}$. Since $\alpha(0+)=0$, we have

where

$$
\begin{aligned}
\int_{0}^{1} e^{x y} y^{\rho} d \alpha(y) & =\lim _{\epsilon=0} \int_{\epsilon}^{1} e^{x y} y^{\rho} d \alpha(y)=\lim _{\epsilon=0} \int_{0}^{-\log \epsilon} e^{x e^{-u}} e^{-\rho u} d\left[-\alpha\left(e^{-u}\right)\right] \\
& =\int_{0}^{\infty} e^{-\rho u} d \beta(u)
\end{aligned}
$$

$$
\begin{aligned}
& \beta(u)=\int_{0}^{u} e^{x e^{-t}} d\left[-\alpha\left(e^{-t}\right)\right] \\
& \beta(0)=0 .
\end{aligned}
$$

Since the function $-\alpha\left(e^{-u}\right)$ is a non-decreasing function of $u$, it follows that $\beta(u)$ is also non-decreasing, and an appeal to Theorem 8 gives the desired result.

It only remains to prove the necessity of the condition. Assume then that

* E. W. Hobson, loc. cit., vol. 2, the first theorem on p. 340 . 
${ }_{-\infty} D_{x} \rho f(x)$ is a completely monotonic function of $\rho$ in the interval $0 \leqq \rho<\infty$ for each $x$. Then by Theorem 16 the sequence $f\left(x_{0}\right), f^{\prime}\left(x_{0}\right), f^{\prime \prime}\left(x_{0}\right), \cdots$ is a completely monotonic minimal set. Consequently, by Theorem 17 ,

$$
f(x)=\int_{0}^{1} e^{x t} d \alpha(t)
$$

where $\alpha(t)$ is a non-decreasing bounded function continuous at $t=0$. This completes the proof of Theorem 18.

13. The complex case. We have been dealing thus far with real functions of the real variable. Although certain of our theorems, such as Theorem 8, are in their very nature real function theorems, others are easily extended to include complex functions. Since it is usually desirable to consider Dirichlet series in the complex plane, it is important to make such an extension in the case of Theorem 14 . We begin by making a similar extension of Theorem 13. We prove

THEOREM 19. A necessary and sufficient condition that the function $f(x+i y)$ can be expressed in the form

$$
f(x+i y)=\int_{0}^{\infty} e^{-t(x+i y)} \phi(t) d t,
$$

where $\phi(t)$ is a complex function of the real variable $t$ which is $O\left(e^{c t}\right)$ as $t$ becomes infinite, is that a real constant $K$ should exist such that

$$
\left|f^{(n)}(x)\right| \leqq \frac{K n !}{(x-c)^{n+1}}(x>c ; n=0,1,2, \cdots) .
$$

The necessity of the condition is obvious from the inequalities

$$
\begin{aligned}
\left|f^{(n)}(x)\right| \leqq & \int_{0}^{\infty} e^{-x t^{n}}|\phi(t)| d t \leqq K \int_{0}^{\infty} e^{-x t^{n} e^{c t} d t}, \\
& \left|f^{(n)}(x)\right| \leqq \frac{K n !}{(x-c)^{n+1}}
\end{aligned}
$$

To prove the converse, set $f(x)=u(x)+i v(x)$. Then

whence

$$
\left(\left[u^{(n)}(x)\right]^{2}+\left[v^{(n)}(x)\right]^{2}\right)^{1 / 2} \leqq \frac{K n !}{(x-c)^{n+1}},
$$

$$
\begin{aligned}
& \left|u^{(n)}(x)\right| \leqq \frac{K n !}{(x-c)^{n+1}}, \\
& \left|v^{(n)}(x)\right| \leqq \frac{K n !}{(x-c)^{n+1}} .
\end{aligned}
$$


Hence, by Theorem 13, functions $\beta(t)$ and $\gamma(t)$ exist such that

Consequently,

$$
\begin{array}{lll}
u(x)=\int_{0}^{\infty} e^{-x t} \beta(t) d t, & |\beta(t)| \leqq K e^{c t} & (t \geqq 0), \\
v(x)=\int_{0}^{\infty} e^{-x t} \gamma(t) d t, & |\gamma(t)| \leqq K e^{c t} & (t \geqq 0) .
\end{array}
$$

$$
\begin{aligned}
f(x) & =\int_{0}^{\infty} e^{-x t}(\beta(t)+i \gamma(t)) d t \\
f(x+i y) & =\int_{0}^{\infty} e^{-(x+i y) t} \phi(t) d t
\end{aligned}
$$

where

$$
\phi(t)=\beta(t)+i \gamma(t)=O\left(e^{c t}\right) .
$$

The theorem is thus established.

TheOREM 20. A necessary and sufficient condition that the complex function $f(x+i y)$ can be represented by a Dirichlet series convergent in the half-plane $x>0$ is that a set of complex constants $a_{1}, a_{2}, a_{3}, \cdots$ and a set of real constants $\lambda_{1}, \lambda_{2}, \lambda_{3}, \cdots$,

$$
0 \leqq \lambda_{1}<\lambda_{2}<\cdots, \lim _{k=\infty} \lambda_{k}=\infty
$$

exist of such a nature that to every positive number $\epsilon$ and to every integer $k$ there corresponds a number $M$ independent of $n$ and of $x$ such that

$$
\begin{aligned}
\left|\frac{d^{n}}{d x^{n}}\left\{\left[f(x)-\sum_{m=1}^{k} a_{m} e^{-\lambda_{m} x}\right] x^{-1} e^{\lambda_{k+1} x}\right\}\right| & \leqq \\
& \frac{M n !}{(x-\epsilon)^{n+1}} \\
& (x>\epsilon ; n=0,1,2, \cdots) .
\end{aligned}
$$

To prove this set $f(x)=u(x)+i v(x)$ and $a_{n}=\alpha_{n}^{\prime}+i \alpha_{n}^{\prime \prime}$. Assume first that $f(x+i y)$ may be expanded in a Dirichlet series

Then

$$
f(x+i y)=\sum_{m=1}^{\infty} a_{m} e^{-\lambda_{m}(x+i y)} .
$$

$$
\begin{aligned}
& u(x)=\sum_{m=1}^{\infty} \alpha_{m}^{\prime} e^{-\lambda_{m} x} \\
& v(x)=\sum_{m=1}^{\infty} \alpha_{m}^{\prime \prime} e^{-\lambda_{m} x}
\end{aligned}
$$

and both series converge for $x>0$. Then by Theorem 14 we have 


$$
\begin{aligned}
& \left|\frac{d^{n}}{d x^{n}}\left\{\left[u(x)-\sum_{m=1}^{k} \alpha_{m}^{\prime} e^{-\lambda_{m} x}\right] x^{-1} e^{\lambda_{k+1} x}\right\}\right| \leqq \frac{M^{\prime} n !}{(x-\epsilon)^{n+1}}, \\
& \left|\frac{d^{n}}{d x^{n}}\left\{\left[v(x)-\sum_{m=1}^{k} \alpha_{m}^{\prime \prime} e^{-\lambda_{m} x}\right] x^{-1} e^{\lambda_{k+1} x}\right\}\right| \leqq \frac{M^{\prime \prime} n !}{(x-\epsilon)^{n+1}}
\end{aligned}
$$

Combining these two inequalities and setting $M=M^{\prime}+M^{\prime \prime}$ we have (13.1). Conversely if (13.1) holds, then the inequalities (13.2) and (13.3) hold if $M^{\prime}=M^{\prime \prime}=M$. It follows by Theorem 14 that $u(x)$ and $v(x)$ are expressible in real Dirichlet series convergent for $x>0$,

$$
\begin{aligned}
& u(x)=\sum_{m=1}^{\infty} \alpha_{m}^{\prime} e^{-\lambda_{m} x} \\
& v(x)=\sum_{m=1}^{\infty} \alpha_{m}^{\prime \prime} e^{-\lambda_{m} x}
\end{aligned}
$$

That is, the series

$$
f(x+i y)=\sum_{m=1}^{\infty} a_{m} e^{-\lambda_{m}(x+i y)}
$$

converges in the half-plane $x>0$. This completes the proof of the theorem. The statement of the theorem could easily be altered so as to deal with an arbitrary half-plane of convergence.

Bryn Mawr College,

Bryn MaWr, Pa. 